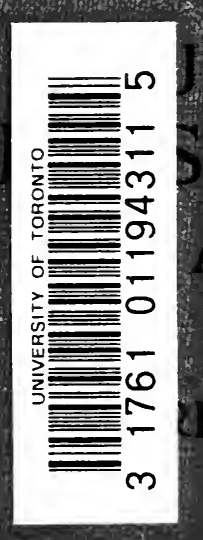

URIES OF THE

NOSE, THROAT, AND EARS

MAJOR

EW MAITLAND RAMSAY

RA.M.C. (T)

MAUOR

O. DUNDAS GRANT RA.M.C. $(V)$ $\rightarrow-4 x^{2}$

CAPT.

H. AWSON WHALE

$$
\text { RAM.O.(I) : }
$$

CAPT

OHARLES ERNEST WEST RAM.C.(I) ?

OXFORD

\title{
WAR PRIMERS
}





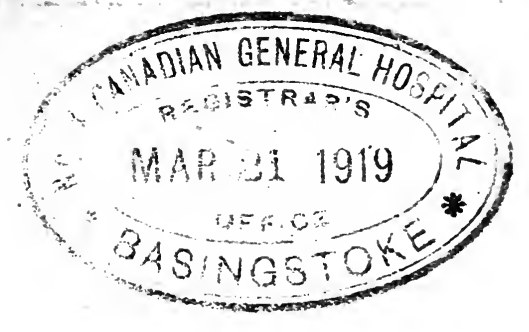


Digitized by the Internet Archive in 2007 with funding from Microsoft Corporation 
INJURIES OF THE EYES, NOSE, THROAT, AND EARS 
PUBLISHED BY THE JOINT COMMITTEE OF HENRY FROWDE AND HODDER \& STOUGHTON AT THE OXFORD UNIVERSITY PRESS WAREHOUSE FALCON SQUARE, LONDON, E.C. 


\section{OXFORD WAR PRIMERS}

\section{INJURIES OF THE}

\section{EYES, NOSE, THROAT}

\section{AND EARS}

BY

ANDREW MAITLAND RAMSAY, M.D., F.R.F.P.S. (Glasgow) OPHTHALMIC SURGEON, ROYAL INFIRMARY, GLASGOW MAJOR R.A.M.C. (T.F.)

J. DUNDAS GRANT, M.D., F.R.C.S. (ENG.) LATE MAJOR R.A.M.C. (POST OFFICE RIFLE VOLUNTEERS); KING GEORGE HOSPITAL, LONDON LORD KNUTSFORD'S SPECIAL HOSPITAL FOR OFFICERS

H. LAWSON WHALE, M.D. (Саmb.), F.R.C.S. (Eng.) CAPTAIN R.A.M.C. (T.F.); FORMERLY NO. I3 GENERAL hOSPITAL, BRITISH EXPEDITIONARY FORCE, OVERSEAS; THE COUNTY OF LONDON WAR HOSPITAL, EPSOM

\section{AND}

CHARLES ERNEST WEST, F.R.C.S. (ENG.)

AURAL SURGEON TO AND LECTURER ON AURAL SURGERY AT ST. BARTHOLOMEW'S HOSPITAL CAPTAIN R.A.M.C. (T.F.)

\section{LONDON}

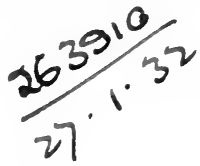

HENRY FROWDE Oxford University Press
HODDER \& STOUGHTON Warwick Square, E.C. 
PRINTED IN ENGLAND

AT THE OXFORD UNIVERSITY PRESS 


\section{CONTENTS}

\section{INJURIES OF THE EYES}

CHAPTER

PAGE

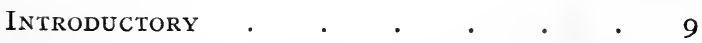

I. Contusion or Concussion Injuries . - i I

II. Penetrating Wounds of the Eyeball • 23

III. Foreign Bodies in the Eyeball • • • 33

IV. Degenerative Changes occurring in Eyes after Perforating Wounds . • . 43

V. Burns and Explosion Injuries . • • 46

VI. Sympathetic Ophthalmia • . $\quad .52$

VII. Amblyopia due to Traumatic Neurosis a 62

VIII. Ocular Signs and Symptoms accompanying HEAD INJURIES INJURIES OF NOSE AND THROAT

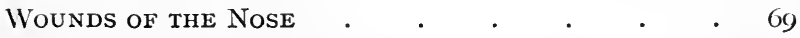
Wounds of the Throat . . . . . . . 90

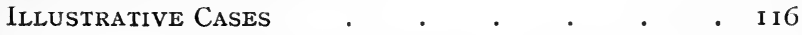

\section{INJURIES OF THE EARS}

The Pinna

The External Auditory Meatus • . . 148

Membrana Tympani and Tympanic Structures • 150

The Bony Labyrinth . . . . . . . I5I

The Mastoid . . . . . . . . $\mathrm{I}_{52}$

DEAFNESS . $\quad . \quad$. $\quad . \quad$. 152

INDEX . $\quad . \quad+\quad \cdot \quad \cdot \quad \cdot \quad$ - $\quad$ I 57 



\section{INJURIES OF THE EYES}

\section{BY}

\section{ANDREW MAITLAND RAMSAY \\ M.D., F.R.F.P.S. (GLASGOW)}

OPHTHALMIC SURGEON, ROYAL INFIRMARY, GLASGOW MAJOR R.A.M.C. (T.F.) 



\section{INJURIES OF THE EYES}

\section{INTRODUCTORY}

In modern warfare eye injuries are of very frequent occurrence, but, whatever their cause-bullet from rifle or shrapnel shell, high explosive shells, hand grenades, foreign bodies thrown up by explosion (stones, earth, glass, chips of metal, \&c.), boiling pitch, petrol or other inflammable substance, or poisonous gases-it may be taken that they differ merely in degree and not in kind from those met with in ordinary civil life. The principles that underlie treatment are, therefore, the same in both instances. From the severity of most of the cases that occur under active service conditions, and the rapidity with which they become septic, there must often be little chance of saving the eye ; and, even though the patient may be attended to at the earliest possible moment, it is obvious that little can be done on the field except to render first aid by removing any foreign body from the conjunctival sac, carefully applying an aseptic compress and bandage, and, if pain be acute, administering a hypodermic injection of morphia.

Unless the eye be hopelessly damaged from the very outset, conservative treatment should be adopted; and all indiscriminate probing for foreign bodies must be deprecated. When the wound is quite recent, the application of antiseptics to the skin of the lids will do good, but the eye itself should be douched only 
with normal sterile saline solution; while, if there be reason to fear infection from tetanus germs, a prophylactic dose of antitetanic serum ought to be given at once. If it be possible, any prolapse of the uveal tract should be carefully excised to prevent its becoming entangled in the wound, which should thereafter be covered completely by a flap of conjunctiva. In every case, after first aid has been given, the aim should be to get the patient, with as little delay as possible, to one of the base hospitals where the necessary appliances and means of special treatment will be available. The sooner the patient can be placed under the care of an ophthalmic surgeon the better. First-aid treatment on the lines just indicated will do much to lessen the three chief complications to be feared in war injuries-sepsis, tetanus, and sympathetic ophthalmia; and it is to be hoped that the pages that follow may help those who, though not experienced in ophthalmic work, will, of necessity, require to deal with eye injuries in warfare.

The subject may best be dealt with under the headings of-

I. Contusion or Concussion Injuries.

2. Penetrating Wounds.

3. Wounds complicated by the Presence of a Foreign Body in the Eyeball.

4. Burns and Explosion Injuries.

5. Sympathetic Ophthalmia.

6. Amblyopia due to Traumatic Neurosis.

7. Ocular Signs and Symptoms accompanying Head Injuries. 


\section{CHAPTER I}

\section{CONTUSION OR CONCUSSION INJURIES OF THE EYEBALL}

THE eyeball is a semi-fluid mass enclosed by membranes possessing little elasticity, consequently when it is struck by a blunt object there may simply be concussion of the whole globe; a portion of its surface may for the moment be flattened; or, if the blow be severe enough, the containing walls may give way and allow the contents more or less completely to escape.

Naturally, then, such injuries divide themselves into those where the full force of the violence falls on the lids and surrounding parts, and those where the eye itself is damaged.

Injuries to the Eyelids.-The simplest and most familiar example of an accident of this kind is a black eye-ecchymosis of the eyelids; and though, in warfare, one would probably not trouble much about such an injury, treatment may sometimes ease discomfort. The swelling and discoloration may be kept in check by the application of cold and a pressure bandage. Nothing is more efficacious than a pocket handkerchief dipped in cold water and tied tightly over the eye. The application of leeches is not to be recommended, neither is puncture of the skin; and the practice of sucking out the effused blood is objection- 
able, and not without danger: I have known it cause a chancre of the eyelid.

Injuries to the Orbit.-When the injury is more severe, the bones at the inner side of the orbit may be fractured, and communication with the frontal and ethmoidal sinuses opened up, thereby allowing air to enter the surrounding cellular tissues-emphysema of the eyelids. This is usually accompanied by epistaxis, and the nature of the soft swelling is readily distinguished by its crackling to the touch, and increasing rapidly in size when the patient blows his nose. The best method of treatment is the application of a pressure bandage; and the patient should also, of course, be warned not to blow his nose.

Blows on the frontal, or on the malar, region are sometimes followed by blindness, the explanation being that such injuries frequently cause fracture through the optic foramen. The optic nerve is thus torn or compressed, and the vision immediately extinguished. Accidents leading to this result may occur in many different ways; the loss of sight in the affected eye is sudden and complete. After an injury of this kind the general symptoms of concussion first occupy attention, and it is usually several days at least before any definite opinion can be formed regarding the state of the eyes. Not till after the patient has recovered consciousness is any complaint made of loss of sight. For some days after the accident there is nothing abnormal in the external appearance, and, although the blindness is absolute, the ophthalmoscope reveals no pathological changes in the fundus 
oculi. The reaction of the pupil, however, shows that the optic nerve has been injured ; for although the consensual reflex is present, and the pupil of the blind eye may respond to the stimulus of light, yet the contraction is only partial and momentary, and full dilatation occurs while the light is still being held in front of the eye. The serious nature of the condition cannot be misunderstood, and in a few weeks it is easy, by means of the ophthalmoscope, to detect complete atrophy of the optic nerve. In such a state of matters treatment is obviously impossible. Even though the skiagrams taken demonstrate an obscure line of fracture, the $\mathrm{X}$-rays are not of so much assistance in diagnosis as one would imagine. If it were possible at first to detect the real nature of the injury it might be practicable to open into the orbit by removing its outer wall as recommended by Krönlein, and, when the optic nerve has been thus exposed, to relieve the pressure, or to reunite the torn ends.

After a blow on the head it occasionally happens that there is complete paralysis of all the muscles of the eyeball-ophthalmoplegia externa and interna. In such a case the lesion is at the sphenoidal fissure through which the third, fourth, and sixth nerves, and the ophthalmic division of the fifth, enter the orbit ; and, in addition to the motor paralysis, there is also anæsthesia of the parts supplied by the ophthalmic division of the trigeminus. When the nerves are simply compressed by blood-clot, or by inflammatory exudation, more or less complete recovery may be expected as absorption takes place; 
but when the bones are fractured and the nerves torn, the paralysis remains permanent. Diplopia is therefore a frequent symptom after such injuries, and in most cases it is well at the outset to give a guarded prognosis.

Injuries to the Globe.-When an eye is struck, the patient generally experiences severe pain, and may for the moment feel stunned. Examination usually reveals abrasion of the cornea, injection of the conjunctiva, congestion of the iris, contraction of the pupil, and more or less dimness of sight. The pupil does not readily yield to atropine, which may have to be instilled several times before full dilatation is produced. Even then the expansion is often irregular, and it is characteristic that the shape frequently becomes oval before assuming the natural circular form. The eye waters, and is so intolerant of light that it may be difficult to make an ophthalmoscopic examination; but if this be possible, it is, as a rule, seen that the details of the fundus are indistinct, as if viewed through a cloud of smoke. If, however, the injury has been more severe, the optic disk shows clear signs of congestion, and between it and the macula the retina presents a greyish swollen appearance. Berlin has described this condition under the name of commotio retinæ, and has demonstrated that, in addition to œdema of the retina, there are also minute hæmorrhages between the choroid and sclerotic.

These instances of concussion of the eyeball differ only in degree, and, although spontaneous recovery in a few days is the rule, it is well not to trust to a 
purely expectant line of treatment, but at once to adopt antiphlogistic measures. As soon as possible after the wound, put the patient to bed, instil atropine, leech the outer canthus freely, apply a cold compress, and administer a purgative.

Hæmorrhage.-In blows of much severity-when, for example, a bullet enters the orbit, even although it does not rupture the globe-the symptoms just described as due to concussion are complicated by hæmorrhage, which may be sub-conjunctival or intraocular.

Sub-conjunctival Hæmorrhage.-Sub-conjunctival hæmorrhage may be limited to the spot where the eyeball was struck, or, as sometimes happens, may completely cover the sclerotic. The effused blood forms a clot, visible through the transparent conjunctiva, more or less completely hiding the sclerotic, and bounded in front by the corneal limbus. Now and then, however, these limits are overstepped, and blood finds its way for a short distance into the substance of the cornea. Gradually the clot becomes absorbed, passing through varying shades of brown, green, and yellow, until the sclerotic again becomes visible. The time required for the disappearance of the blood varies from a few days to several weeks, but in all cases in which the hæmorrhage has been extensive a yellowish staining of the white of the eye remains for a much longer time. When sub-conjunctival ecchymosis occurs immediately after a wound it is of very little significance, and nature alone will do all that is required for its removal. If, however, the 
hæmorrhage makes its appearance after a lapse of several days the prognosis is very serious, because it is then one of the most trustworthy indications of fracture through the anterior fossa of the skull. Th: sign is, therefore, of great value as indicating a grav condition which may be followed by death, so that it is always very important in every case of injury to the head to note whether sub-conjunctival ecchymosis be present, and to ascertain if possible whether it appeared immediately after the accident, or not for several days.

Intra-ocular Hæmorrhage.-Intra-ocular hæmor rhage is always serious, though the gravity of the condition is much less when the blood is in the aqueous, than when it is in the vitreous, chamber. It may indicate not only rupture of blood-vessels but also rupture of some of the internal struc tures of the eyeball. As long as the hæmorrhag, persists, it is, of course, impossible to say how mucl damage has been done, and it is only after the clot disappears that the true nature of the injury becomes apparent. If the effusion be confined to the aqueous, the bleeding comes from the vessels of the iris or ciliary body; but if it be into the vitreous, it may come also from the vessels of the choroid or retina.

From Iris.-The iris frequently suffers. As a result of a contusion both iris and ciliary body may be paralysed, and, though occasionally either or both of these conditions may persist, yet, in most instances, the dilatation of the pupil and the loss of the power of 
accommodation pass away in a few days. Structural lesion, whether it occur at the pupillary border or at the ciliary margin, is permanent, although in its islighter forms it does not necessarily interfere with sight. A small clot arising from simple rupture of blood-vessels may easily be mistaken for a structural tear-an error that time will soon show, as the blood will quickly disappear. Lacerations of the pupillary border, so small that they. can be detected only by careful examination, give rise to permanent dilatation of the pupil, and if a distinct rent be visible the pupil will also be irregular in outline. When the iris is separated from its ciliary attachment (iridodialysis) the point of lesion is marked by a black crescent, and the pupil loses its rounded form, being flattened on the injured side. If the aperture be at first small, it will, in course of time, become less, but if it be large it will always remain visible, and when light is transmitted to the eye by the ophthalmoscope a red reflex is obtained through the rupture as well as through the pupil. In severe cases there may be more than one lesion, and occasionally the whole iris is separated, and may either lie loose in the anterior chamber, or be extruded from the eyeball, giving rise to the condition known as traumatic aniridia.

From Choroid.-Hæmorrhage from the vessels of the choroid is always a very serious matter, because the blood will be effused eitherbetween the sclerotic and the choroid or between the choroid and the retina. The former condition involves separation of the two structures concerned, and the latter more or less extensive

R.G.W. 
detachment of the retina. The history of the accident, the suffering of the patient, the appearance presented by the eye, and the diminution of the intra-ocular tension, would lead one to suspect rupture of the choroid even although the presence of the blood render it impossible to see the lesion. When, after a time, longer or shorter according to the size of the bloodclot, the media become sufficiently transparent to permit the fundus to be seen, there will be found in the choroid, usually to the outer side of the papilla, one laceration, possibly more than one. The rupture shows itself as a narrow streak, thick in the middle, pointed and sometimes branched at the extremities, and bordered by pigment, the white colour being due to the fact that the sclerotic is visible through the tear. As time goes on the inflammatory symptoms subside, and the outline of the lesion is more sharply defined, while in many instances the posterior pole of the eye becomes distinctly pigmented.

From Retina.-Rupture of the retinal vessels through violence is not nearly so common; and when it does occur, the hæmorrhage takes place, as a rule, between the retina and the hyaloid, giving rise to a large disk-shaped clot situated, in most instances, in front of the macular region. That the retina itself is not seriously implicated in this bleeding is clear from the fact that, although the patient may not be able to distinguish any object, yet when he looks at a gas or candle flame it appears red, this being due to the blood coloration. Such clots become, in most cases, gradually absorbed. and, if the eye has not been 
otherwise injured, the sight will, in course of time, be completely restored.

Where detachment of the retina is the result of violence, the prognosis is much more favourable than when it is due to disease; but under any circumstances the condition is always most serious. If the detachment is localized it will be distinctly visible on ophthalmoscopic examination, and its extent may be mapped out by the restriction of the visual field as measured by the perimeter. In such cases something may be accomplished by suitable treatment, but if the separation be complete there is no hope of recovery of sight.

Dislocation of the Lens.-A contusion so severe as to produce the injuries just described will also almost certainly cause rupture of the zonule and dislocation of the lens. The displacement may be partial or complete. In the former case the lens remains in the posterior chamber, its black outline being readily visible on ophthalmoscopic examination, and if the vitreous be fluid it is seen to swing up and down, and the iris, deprived of its support, trembles with every movement of the eye. In complete dislocation, the lens may be forced into the vitreous, and may lie at the bottom of the chamber quite removed from the line of vision. In other cases, more especially if it be small, it escapes through the pupil and falls into the aqueous. In this situation it acts like a foreign body, and may give rise to much trouble by setting up secondary glaucoma. After dislocation the lens may remain transparent,. but more usually it becomes cataractous, and in old- 
standing cases undergoes calcareous degeneration, its bulk being so much reduced that it slips backwards and forwards through the pupil without any difficulty.

Rupture of the Globe.-When the injury is more severe, the tissues of the eyeball are ruptured, and the lens escapes beneath the conjunctiva. The sclerotic usually gives way at its upper or inner aspect, just beyond the corneo-scleral border, where it is naturally weak owing to the presence of the canal of Schlem. It is easy to distinguish the lens as a little swelling covered by conjunctiva. At the site of the rupture the iris seems wanting, owing to its having been driven back into the vitreous at the time the lens escaped. Here, as in the other instances that have been described, the true nature of the injury is at first often obscured by the occurrence of hæmorrhage, followed later by considerable inflammatory exudation. Although the upper and inner aspect of the globe is the usual site for rupture of the sclerotic, the eye may, through direct violence, be burst at any point. Where the blow is a very severe one the rent in the tunics is so large that the clear structures escape, and the choroid and retina prolapse through the wound. The globe becomes filled with blood, which may infiltrate the substance of the cornea and form a thin layer in front of the iris, giving rise to the appearance of a pupil although no pupil is there. There is always great chemosis of the conjunctiva, and swelling and discoloration of the lids.

Treatment of Injuries of Globe.-In the treatment of these severe conditions one must neces- 
sarily be guided by circumstances, and good results can follow only if the structural damage has not been extensive. A clot in the aqueous is readily absorbed, but one in the vitreous is much more persistent. It breaks up in course of time, but traces of it remain in the form of little black flakes which disturb sight. It may, however, as a general rule, be said that the antiphlogistic line of treatment, mentioned at p. I5, is applicable to all cases in which there is any chance of recovery, and that, in addition, special complications require special remedies. Traumatic separation of the retina is most likely to be benefited by prolonged rest in bed, by the use of the pressure bandage, and by sub-conjunctival injections of a solution containing one per cent of dionine and eight per cent of pure chloride of sodium in I in 2,000 bicyanide of mercury. If the site and character of the detachment be favourable the sub-retinal fluid should be withdrawn by scleral puncture.

The lens should, in cases of dislocation, be extracted as soon as possible. It is easy to remove it from the aqueous chamber, provided it can be kept there by contracting the pupil with eserin till the operation be completed; but, should there be any chance of its slipping back into the vitreous chamber, it should first be fixed by passing a needle behind it, before the extraction is begun. When, however, it is floating free in the posterior chamber operative interference is undesirable unless there be some special reason for it. Sub-conjunctival dislocations are easily dealt with by slitting the conjunctiva over the lens and allowing the 
latter to escape; but the operation should never be attempted till several days after the accident, in order that the wound in the sclerotic may close before the conjunctiva is disturbed. This delay prevents any risk of the lens slipping back through the wound, and diminishes the danger of septic infection. When an eyeball is burst, enucleation is the best possible treatment; and the sooner the operation is performed the better, in order to anticipate the onset of inflammation and suppuration, and so to save the patient much needless suffering. 


\section{CHAPTER II}

\section{PENETRATING WOUNDS OF THE EYEBALL}

Penetrating wounds of the eyeball may be divided into lacerated, incised, and punctured. The first are the result of a blow which causes rupture of the sclerotic, and have already been described. When the eye is penetrated by a bullet it is ripped up into numerous leaves and its contents eviscerated more or less completely. Colonel Lister has likened the appearance it presents to "the corolla of a very much faded flower'. The most distressing cases are those where vision has been destroyed by a bullet which has passed transversely through both orbits. In the present war an unusually large number of soldiers have been blinded in this way, chiefly owing to the activity of the sniper. Incised and punctured wounds are inflicted by a knife, a pair of scissors, a fork-prong, a pen, a piece of glass, a chip of metal, a bullet, \&c. Where the injury is due to the bursting of a bottle, or to any accident of like nature, the lids are nearly always severely implicated, and, swelling up, conceal the globe. In such cases great care must be taken not to overlook a wound of the cornea and sclerotican error in diagnosis which might obviously be followed by most serious results. Should the globe be perforated, the great risk is that whatever caused the 
wound may at the same time have carried infection with it. Aseptic wounds are to be found only after carefully performed surgical operations: all other wounds may be regarded as septic, and though the injury may, in a case of puncture, be very slight, so slight indeed as to be hidden by sub-conjunctival ecchymosis, yet should micro-organisms have been conveyed into the interior of the ball, the eye may in a few days be entirely destroyed by suppuration. The difficulty of diagnosing these seemingly trivial cases will be considerably lessened if care be taken to examine the tension of the globe: whenever there is an opening in the cornea or sclera the intra-ocular tension is diminished.

Incised Wounds.-Incised wounds, usually self-evident in themselves, are, besides, accompanied by prolapse of the iris, ciliary body, or choroid; by injury to the lens; and by escape of the vitreous. If they be extensive, and more particularly if the ciliary region be involved and the escape of vitreous be large, the eye will almost certainly be lost, because, even although the wound heal, there is always subsequent inflammation of the uveal tract, and this leads to shrinking of the globe, and possibly to sympathetic inflammation. Indeed the chances of preserving a serviceable eye after a penetrating wound will depend very largely upon the site and extent of the injury, the amount of the loss of vitreous, and the risk of septic infection.

Corneal Wounds. - Wounds of the cornea admit, as a rule, of a more favourable prognosis than those involving the sclerotic, not because the cornea is less 
casily infected, but probably because the aqueous humour on escaping washes the micro-organisms away from the lips of the wound. The progress of the case is governed, however, more by the depth of the injury than by its surface extent; and hence it is that puncture wounds are always so disastrous, for they not only involve the cornea or sclerotic, but they generally also implicate the iris, ciliary body, and lens. Simple wounds of the cornea heal very rapidly. It is of the utmost importance, therefore, that the lips of a corneal wound should come into accurate apposition, but this is frequently prevented by prolapse of the iris. Should this have happened, the fate of the eye depends, in most instances, on the skill with which the prolapse is treated.

Prolapse of the Iris.-If the prolapse be recent, it should be carefully excised. The iris should be firmly caught by forceps, and, while it is held slightly on the stretch, should be cut through by a single snip of curved scissors, the blades of which are pressed backwards against the eyeball. If the iris be kept tense in this manner the cut edges almost always retract perfectly within the aqueous after the prolapse is excised, and it is most important that no part should remain adherent to the wound in the cornea. After the excision, the lips of the latter ought to be brought into accurate apposition by means of a small spatula, and, the eye having been thoroughly cleansed by copious douching with hot boracic solution, or with sterile saline solution, the wound should be covered by a flap of conjunctiva, which not only holds the lips together and thereby 
stimulates healing, but also acts as a great protection against septic infection.

If, however, more than forty-eight hours have elapsed before surgical treatment begins, the prolapsed iris will be firmly sealed to the corneal wound by inflammatory exudation, and before any attempt at excision is made these adhesions must be carefully broken down. If they are so firm as to be separated only with difficulty, the surgeon must allow his experience to guide him as to whether he should persevere in his attempts or should leave the case to nature. In the majority of instances the latter is probably the more prudent course, for very soon the base of the prolapse becomes surrounded by a line of white inflammatory exudation, which by and by contracts and forms a firm constriction. At a later period its surface is coated with new-formed tissue, and it is, by cicatricial changes, flattened against the cornea and held there securely under a white scar, towards which the pupil is displaced. That is nature's method of cure, and on the whole the results are satisfactory. Indeed, should several days have passed before surgical treatment can be begun, and the iris be glued by exudation to the lips of the wound, interference might open a path for septic infection.

There are, however, cases where the prolapse is so large that it is dangerous to leave it alone. Under such circumstances it is probably best to wait until the base of the prolapse is sealed firmly to the lips of the corneal wound and all signs of acute irritation have passed away, and then by means of a cautery heated 
to a dull red heat carefully to burn down the prolapse to the level of the cornea, taking great care not to make an opening into the anterior chamber. After the operation the wound should be covered by a flap of conjunctiva.

It happens, though rarely, that, when the cornea is injured, eyelashes are driven into the eye and imprisoned in the anterior chamber. Their presence in this unnatural position tends to keep up inflammation, and may form the starting-point for the development of an implantation cyst of the iris.

Wounds of Sclerotic.-While simple wounds of the cornea, as has been already said, heal very rapidly, those of the sclerotic, on the other hand, do not heal of themselves. The sclera is passive, and union is effected by reparative changes in the episcleral tissue, in the conjunctiva, and in the choroid. The gaping which always occurs should be dealt with not by suture of the sclerotic itself-an operation both difficult and dangerous-but bysuture of the conjunctiva. Wounds that implicate the choroid and retina-as so frequently happens-may, under favourable circumstances, heal without trouble; but they are often followed by detachment of the retina and by inflammatory changes which terminate in shrinking of the globe. An injured lens is always a serious complication, for it rapidly becomes cataractous, and, by swelling and acting as a foreign body, renders the original mischief much more serious. In order to diminish the risk of cyclitis, the cataract should be washed out by copious irrigation with warm and sterile saline solution. The 
irrigator must be rendered thoroughly aseptic by being boiled, and then, the point having been carefully introduced into the aqueous through the wound in the cornea, the stream of fluid is directed in such a way as to pass inside the capsule. Should the injury be extensive and the eyeball collapsed through loss of vitreous, the globe may, on rare occasions, be saved by filling it up with sterile saline solution; but probably in the majority of cases the wisest plan is to enucleate at once, so that the patient may be saved much useless suffering, and the risk of sympathetic inflammation be avoided.

Treatment of Recent Wounds.-In a recent wound, when the eye does not contain a foreign body, cleanse the conjunctival sac thoroughly with a hot saturated solution of boracic acid, or with physiological saline solution, carefully clear away any prolapse of iris or ciliary body, irrigate the anterior chamber, wash out the lens if it be cataractous, and apply a gelatine disk containing ten per cent collargol over the wound, which is then covered by a flap of conjunctiva. All that remains to be done thereafter is to instil a drop of a two per cent solution of atropine, to bandage the eye carefully, and to put the patient to bed. To relieve pain, which is often very severe, an ice-bag should be applied over the bandage, and one-sixth of a grain of morphia injected beneath the skin of the temple. The immediate danger is the occurrence of inflammation of the iris and ciliary body, and in all cases this should be anticipated by careful asepsis, by the use of atropine, and 
by a sharp calomel purge. A five per cent solution of dionine in addition to the atropine is to be recommended. It quickly relieves the deep-seated pain, and hastens the absorption of lenticular matter.

Later Treatment.-After the wound has healed, the opaque capsule of the lens, to which the iris is always more or less adherent, blocks the pupil and interferes seriously with vision. It is usually necessary, therefore, to perform a capsulotomy. An incision is made in the cornea with a keratome, and a pair of Carter's or De Wecker's scissors introduced through the opening into the anterior chamber. The blades of the scissors are opened, and one passed behind and the other in front of the constricting band of capsule, which is then divided. If the operation be successful, the iris at once springs open to form a pupil. Each case must be judged on its merits, and as a rule the principal incision must be made where previous examination has shown the iris to be most tightly stretched. The opening in the capsule can be made larger by one or more cross-cuts. Great care must be taken to avoid the escape of vitreous, and, if the patient be nervous, it is, as a rule, safest to administer a general anæsthetic, or preferably to give omnopon-scopolamine intramuscularly an hour and a half before the operation.

Treatment of Infected Wounds.-When there is infection at the time of the injury, pain is always severe and is decp-seated and throbbing in character, the lips of the wound become infiltrated, the conjunctiva œdematous, and the iris discoloured. In such circumstances the suppurative process may be 
held in check by free irrigation of the conjunctival sac with hot sterile saline solution. This mechanical cleansing washes away all discharge and diminishes the number and the activity of the micro-organisms. The ocular congestion is lessened, and pain relieved, by snipping the chemosed conjunctiva with scissors, and encouraging the bleeding by douching with warm salt solution. A collargol disk ought to be placed in the conjunctival sac every two or three hours, and the first assurance that the infective process is being overcome is given when the collargol gelatine is seen to cling to the lips of the wound. The eye must be kept covered by antiseptic fomentations, and the pain relieved by the judicious use of hypodermic injections of morphia. The patient ought to be kept in bed, well fed, and skilfully nursed.

A cataractous lens is one of the best culture media for micro-organisms, and so, when the interior of the eyeball becomes infected, the process of suppuration goes on unchecked by the treatment just described. The pain persists and is always characterized by periods of exacerbation during the night. The eyeball is exceedingly tender to the touch, the iris is intensely inflamed, and, if the lens retains its transparency, a yellowish reflex is occasionally seen through the pupil. If, on the other hand, the lens has been injured, the purulent exudation imparts a yellow colour to the opaque lenticular substance. Pus very soon appears in the anterior chamber, and the condition is extremely critical. Even in such desperate circumstances an attempt ought always to be made 
to save the eye. The wound in the cornea must be opened up, and the cautery applied thoroughly to its edges. The anterior chamber should be irrigated freely with saline solution, and any pus completely washed away. A few drops of a twenty-five per cent solution of argyrol ought then to be injected carefully into the interior of the eyeball, or a little rod of gelatine impregnated with collargol may be introduced into the aqueous chamber, where it gradually dissolves. My experience is more favourable to the use of argyrol and collargol in this way than to that of iodoform.

In most instances, however, the infective action has penetrated to a point too deep to be reached by any antiseptics, and the probability is that the eye will be lost from panophthalmitis, or from chronic iridocyclitis. Whenever, therefore, it is evident that sight is to be lost it is by far the wisest course to enucleate at once. If there be acute suppuration of all the tissues of the globe an element of danger attends enucleation, for there is then a risk that the operation may be followed by purulent meningitis. Under such circumstances it is probably wiser to eviscerate than to enucleate. In these cases Colonel Lister has recently advised, in order to get the rapid healing which usually follows enucleation, and at the same time to avoid the risk of meningitis, that after the cornea has been removed the contents of the globe should be thoroughly eviscerated, and after the ocular muscles have been divided the sclerotic should be cut 'far back, leaving a frill of about four or five millimetres in radius round the intact optic nerve'. 
Prothesis.-Ophthalmic surgeons have suggested many means whereby the deformity produced after the removal of an eyeball may be lessened, and a more natural appearance given to the prothesis. Mules suggested evisceration and the implantation of a glass or silver ball in the sclerotic. The cosmetic results following the successful performance of this operation leave little to be desired; but it has the three great disadvantages that the whole of the eyeball is not removed, that there is a tendency for the artificial vitreous to escape from the sclerotic, and that healing after the operation is often accompanied by much inflammatory œedema of the orbital tissues and by very severe suffering for several days. In order to get rid of these disadvantages I form a stump for an artificial eye by injecting paraffin into Tenon's capsule. The paraffin simply lies in the tissues and gives rise to no irritation. The advantage is not so much in the greater mobility of the prothesis as in the minimizing of the flat, sunken appearance of the upper lid which nearly always follows simple enucleation.

The laceration of the lids and the infiltration of the tissues of the orbit which necessarily follow septic bullet-wounds cause much scarring and cicatricial contraction of the socket, and such cases will require plastic operations to form new eyelids and enable the patient to wear a glass eye. 


\section{CHAPTER III}

\section{FOREIGN BODIES IN THE EYEBALL}

OwING to the bursting of a shell minute particles of earth, stone, metal, \&c., may be carried through the coats of the eyeball, and the small wounds caused thereby may be completely healed by the time the patient is examined. The doctor may therefore in making a diagnosis of iridocyclitis forget the possibility of a foreign body being present. Nevertheless the latter is probably the most serious factor in the case, because injuries accompanied by a foreign body in the globe are peculiarly liable to be followed by sympathetic inflammation. On the other hand, one ought not to decide at once that an eye containing a foreign body must invariably be enucleated, for I have at present under my care a patient who has carried about a small piece of steel in his right eye for forty years, and it is only within the last few months that he has suffered any inconvenience as regards sight or has had any inflammatory trouble. His left eye has never been affected at all. In this case the particle of steel, though perfectly visible by the Röntgen rays, has become encysted, but the occurrence of inflammation after the lapse of such a long time emphasizes the fact that so long as any foreign body remains within the globe the safety of R.G.W. 
the eyeball cannot be regarded as permanently assured. When a patient complains that he has received an injury to his eye, the doctor should invariably make a careful examination of the conjunctival surface of the upper lid. It is necessary, however, if it be suspected that a foreign body is present, not only to evert the eyelid, but also to make a careful exploration of the cul-de-sac with a small probe. It is all the more needful to bear this in mind, because, though the smallest particle of dust within the eye usually gives rise to intolerable uneasiness, yet larger bodies may remain for weeks in the loose folds of this cul-de-sac, without their presence being indicated by any signs more serious than inflammation accompanied by swelling of the conjunctiva, and puro-mucous discharge. Such an inflammation, however, does not yield to the usual remedies, and subsides only after the removal of the exciting cause.

Particles of dust, \&c., are usually found lying loosely upon the conjunctiva, and are, as a rule, readily removable with the point of an unused toothpick, a camel-hair brush, or the edge of a small spud; but should the foreign body be firmly fixed in the substance of the cornea or of the conjunctiva, the difficulty of its removal is directly proportionate to the depth to which it has penetrated.

Method of removing Foreign Bodies.-In dealing with cases of this description, seat the patient in a good light, with his head firmly supported by the back of a chair, or, preferably, by the hands of an assistant. 
Separate the eyelids with the thumb and index-finger of the left hand, and press slightly backwards, so as to fix the eyeball; then pass the needle or gouge, or preferably a small Beer's knife, behind the foreign body, and lift it from the cornea. Should it have penetrated deeply, and there be danger of pushing it back into the eye, a second and broader needle should be passed into the anterior chamber behind, and with this extra support removal is usually a matter of no great difficulty. If any staining of the cornea remains after the operation it ought to be carefully scraped away, so that the healing process may be hastened. When the substance is embedded in the conjunctiva it should be grasped with a pair of forceps, and the piece to which it is adherent cut out with a pair of curved scissors.

After-treatment.-Removal is in all cases followed by an immediate sense of relief, and, as a rule, nothing more requires to be done, except to bathe the eye with some antiseptic lotion, and, if pain continue, to apply an ointment of cocaine and vaseline, and to adjust a compress and bandage. These should be worn until all signs of irritation have passed away. When, however, in consequence of the foreign body having been long present, or of rough and unskilful attempts having been made without success for its removal, the breach in the corneal surface is slow to heal, the wound should be lightly dusted with equal parts of precipitated iodoform and boracic acid in very fine powder. This treatment stimulates the healing process and counteracts, as far as possible, any 
tendency to suppuration. Acetate of lead should never be used. Lead lotions have enjoyed a welldeserved popularity in the treatment of conjunctival inflammations, but, whenever there is a breach of surface, the acetate is decomposed and a white precipitate is formed. If this be deposited on the cornea, a dense white glaring opacity is the result, and the vision of the eye is permanently impaired.

\section{Penetrating Wounds of the Eye with Retention of a Foreign Body}

It is only very occasionally that the presence of a foreign body can be determined with the ophthalmoscope. In most instances it rapidly becomes concealed by hæmorrhage, or hidden by increasing opacity of the lens, or by an outpouring of inflammatory exudation. It is, therefore, of the first importance that the eye be thoroughly examined at the earliest possible moment after the accident, and the nature of the latter, along with its attendant circumstances, ought always to be carefully and fully elicited. Obviously an eye which has been penetrated by the prong of a fork, or cut by a knife, is not at all likely to contain a foreign body; but, on the other hand, when the accident has been due to a blow from a chip of steel, of glass, or of stone, or where it has been the result of an exploding cartridge, or of a shot-splinter, the risk that the missile has become lodged in the globe is exceedingly great.

Signs and Symptoms.-The appearances presented 
by the eye are also of great value in aiding a diagnosis of the presence or absence of a foreign body. If this be present there is usually a penetrating wound of the cornea or of the sclerotic, the iris is torn, the aqueous stained by blood, and the lens cataractous. It is often remarkable how slight is the suffering immediately after such a severe injury. The patient rarely makes any complaint of pain, but states that the sight is more or less defective. In a few days, however, there is marked congestion of the circumcorneal blood-vessels, and considerable tenderness on pressure over the ciliary region. The degree of inflammatory reaction subsequent to the injury depends not only upon the size and shape of the foreign body, and the position which it occupies within the globe, but also upon the changes which it is capable of originating, as a result either of direct sepsis or of simple chemical reaction between the foreign body and the tissues in which it is embedded. Chips of metal, for example, nearly always set up severe inflammation, whereas splinters of glass, if introduced into the eye aseptically, may produce but little disturbance. Leber has shown that copper is particularly dangerous, even when it is quite free from micro-organisms, for it always sets up destructive inflammation as a result of chemical reaction.

The account of the injury, the position and character of the wound, and the subsequent history of the case, may all afford strong presumptive evidence that the eye contains something extraneous, but this suspicion can be confirmed only by actual demonstration of the 
presence of a foreign body. If the media be transparent the substance may be seen either by the naked eye or by means of the ophthalmoscope, but care should always be taken not to mistake a small blood-clot, or a minute rent in the iris, for a particle of metal. In the majority of instances, however, the media are not transparent at the time the patient seeks advice. If the foreign body be a chip of "iron or steel its presence may be determined by aid of Hirschberg's sideroscope or by bringing a powerful magnet closely up to the eye, when the patient will at once experience sharp pain owing to the metallic particle being attracted from the deeper parts of the eye towards the surface. The Röntgen rays often afford most valuable assistance, for not only do they render it easy to demonstrate the presence of a foreign body whose existence would otherwise be doubtful, but they give still greater assistance in determining its exact position.

Extraction of Metallic Foreign Bodies.-Even after a foreign body has been carefully localized, its extraction from the eyeball is always extremely difficult, unless it be a chip of iron or steel, which can be attracted by a magnet. In every magnet operation the great secret of success lies in prompt intervention. If the case be dealt with within twenty-four hours of the accident the patient should be laid flat on a table with the head and shoulders comfortably supported by pillows. The eye should be thoroughly cleansed, the pupil dilated as fully as possible, and the conjunctiva made perfectly insensitive. A general anæsthetic is necessary only in the case of a very nervous 
or troublesome patient, because an injection of novocaine and adrenaline deep into the tissues of the orbit renders the operation painless.

If the wound be in the cornea an attempt ought to be made to extract thechip of metal through it; but if, as so often happens, it be situated at the corneoscleral junction, or in the sclerotic farther back, it is safer to try to guide the metal from the vitreous chamber into the aqueous, rather than run the risk of injuring the ciliary body and choroid still further, and of disturbing the vitreous by dragging the foreign body through the perforation caused by the wound. Of course every now and again a case occurs in which the cut in the sclerotic is so situated that the splinter may be most readily extracted by enlarging the rent, if necessary, and applying the point of the magnet to its lips; but as a general rule the eye will be damaged less if the magnet be used in such a way as to draw the foreign body to the aqueous chamber along the path of least resistance. If then the point of the magnet be held against the centre of the cornea, and the current gradually increased by means of the rheostat, the patient will, as a rule, suddenly give a sharp cry of pain, and the metallic particle will be seen to pass in front of the lens and to bulge the iris forwards.

If, as sometimes happens, there is difficulty in attracting the foreign body, the operator must exercise patience and apply the current in such a way as to act on the metal by a series of jerks. The splinter reaches the aqueous by passing behind 
the lens and perforating the zonule; and, indeed, provided that the lens has escaped injury at the time the wound was inflicted, it is very seldom injured during a skilful attempt to extract a piece of metal.

It is most important that the field of operation be properly illuminated, so that every movement of the foreign body may be quickly seen and all damage from tearing of the iris prevented. The current ought at once to be shut off as soon as the splinter reaches the aqueous.

The operator must be guided by experience as to further procedure. If the splinter possess sharp edges which have caught the iris, the safest way is to incise the cornea and cut out the piece of iris overlying the metal; but if the iris be not involved an attempt ought to be made to draw the chip gently into the pupil and to keep it there until the anterior chamber is opened. The particle can then be removed without an iridectomy.

In practice it is well to have a Hirschberg's magnet at hand in addition to a giant magnet, because, if the rough edge of a chip of metal becomes entangled in the iris, there is great danger that the large magnet will extract it so rapidly and forcibly that a considerable portion of the iris will be dragged out at the same time. It is better, indeed, after the foreign body has been displaced from the deeper parts of the eye into the aqueous chamber, to complete the operation for its removal with the less powerful instrument.

If, on the other hand, the patient be not seen till 
the wound in the cornea or sclerotic has had time to become firmly closed, the site of the splinter must be determined as accurately as possible, and an incision made through the sclerotic in its immediate neighbourhood. The point of the magnet can then be introduced within the lips of the wound and the metal extracted. After the operation the cut in the sclera must be carefully covered by conjunctiva to prevent infection, and the patient should be kept in bed for at least a fortnight. If no means be at hand for accurately fixing the position of the extraneous substance, the large magnet should be applied to the centre of the cornea and the chip of iron or steel attracted and drawn into the anterior chamber.

Prognosis.-The sooner the foreign body is extracted, the greater the chance of preserving sight; and if the lens has escaped injury, obviously the prognosis is so much the more favourable. Under such circumstances a piece of metal of considerable size may be removed from the vitreous chamber, and the patient be not much the worse.

In many cases, however, in which the operation has been performed with promptitude and success, sight may afterwards be completely lost owing to detachment of the retina, or the globe may in the end be completely destroyed by plastic cyclitis. The eye is indeed damaged irreparably from the first. The shock, the large size of the foreign body, and the great liability of the vitreous to become infected by septic organisms, all contribute to bring about a disastrous result. 
Hirschberg divides foreign bodies according to their weight : the small, weighing from twenty to thirty milligrammes, can usually be successfully extracted; while the medium, weighing from fifty to a hundred and fifty milligrammes, imperil the globe in proportion to their size, and raise the question whether it would not be wiser to enucleate than to make any attempt to save the eye. If the foreign body be one which is not attracted by the magnet, and unfortunately this is specially the case with most of the substances which gain entrance to the globe on the field of battle, all that can be done after accurately localizing its position within the eyeball is to cut down upon it and attempt to extract it with forceps. It is not often, however, that those efforts are successful, and the operation is usually completed by enucleation: indeed under such circumstances the surgeon ought always to take the precaution to have previously obtained the patient's consent to remove the eye if he considers it advisable to do so Failure may sometimes also occur although the foreign body be one which the magnet will attract, for it is not at all unusual for a particle of metal to become so embedded in the ocular tissues as to be completely insulated; and the more minute the particle the greater this danger. 


\section{CHAPTER IV}

\section{DEGENERATIVE CHANGES OCCURRING IN EYES AFTER PERFORATING WOUNDS}

Although it is not always possible to preserve the function of an eye after a perforating injury, or after the successful removal of a foreign body, yet modern surgical technique and the advances of physical science enable the surgeon, greatly to the satisfaction of the patient, to preserve its form. Unfortunately, however, it sometimes happens that after an apparently successful result degenerative changes supervene, and these give cause for much concern regarding the fate of the injured eye and the safety of its fellow. Roughly speaking, these retrograde processes result either in shrinking, or in distension of the injured globe, and of these the former is by far the more frequent.

Degenerative Changes in the Eyeball.-An eye may seem for several weeks to be recovering quite satisfactorily, and the patient may even be able to see with it, but then the cicatrix of the wound begins to retract, while at the same time the sight first becomes weaker and then disappears altogether owing to detachment of the retina. The intra-ocular tension gradually diminishes, the cornea gets flatter and smaller, and the anterior chamber becomes so shallow that the iris at length lies closely against the posterior 
surface of the cornea. The globe loses its spherical shape and assumes a somewhat quadrilateral form, owing to flattening of its walls at the insertion of each of the recti muscles, and the upper eyelid tends to droop and fall in. Hæmorrhage frequently occurs from the vessels of the iris and ciliary body, and the iris becomes much altered in colour from the absorption of blood pigment. All this time there is tenderness on pressure over the ciliary region, the eye readily flushes and waters on exposure, and there may be complaint of sympathetic irritation.

Obviously enucleation is here the proper treatment ; but should the patient (as is sometimes the case) refuse to allow the eye to be removed, there will be recurrent attacks of inflammation, accompanied frequently by much suffering, and lasting for many months. After they subside, the stump, now shrunken to a very small size, may remain quiescent for years; but as long as it remains it is, as a rule, unwise for the patient to wear an artificial eye, because the prothesis may cause irritation sufficient to precipitate the onset of sympathetic mischief. These shrunken globes are the seat of frequent pathological changes; deposits of lime take place in the ciliary body and lens, and the choroid gradually becomes converted into a shell of bone.

Distension of the eyeball, which is more likely to follow a punctured than an incised or a lacerated wound, is due to general inflammation of the uveal tract. The cornea and sclerotic may become uniformly distended, but more usually staphylomata 
form in the ciliary region and at the equator. The vitreous becomes quite fluid, and frequently contains numerous cholesterine and tyrosine crystals-synchysis scintillans - the intra-ocular tension isincreased, there is always liability to the occurrence of pain and inflammation from recurrent glaucomatous attacks, and sooner or later, owing to detachment of the retina, sight is completely lost. Such an eye is not only very disfiguring, but is also a serious menace to its fellow.

Treatment.-It cannot be insisted on too strongly that every eye which is blind and painful ought to be enucleated, for not only is it a constant source of trouble to its possessor, but it may at any time give rise to sympathetic ophthalmitis, and it may, besides, become the seat of a malignant growth. If, however, the patient refuse to have the eyeball removed, the danger of sympathetic mischief may be diminished by dividing the optic and ciliary nerves; and the disfigurement may be lessened by performing the complete keratectomy operation recommended by Panas. 


\section{CHAPTER V}

\section{BURNS AND EXPLOSION INJURIES}

OF all injuries to the conjunctiva, none is more disastrous in its results than a burn, especially one due to the action of any chemical irritant. In all cases of this kind the prognosis must be very guarded, as the action of the chemical extends far beyond its point of application, and what seems at first sight only to have caused a greyish haze over a part of the surface of the cornea will probably by the end of a fortnight have brought about the total destruction of that membrane, and the consequent extinction of vision.

In all cases of burns or scalds of the eyeball the general symptoms are the same, and their severity depends upon the nature of the irritant, the length of time it has remained in contact with the eye, and the extent of the structural injury it has produced.

Symptoms and Signs.-After a burn, from whatever cause, the pain is very severe, and the inflammatory reaction is rapid and violent. The eyelids are usually injured, but whether they are or not they rapidly assume a dusky red colour, and become so swollen that the interior of the eye can be seen only with difficulty. There is chemosis of the ocular and palpebral portions of the conjunctiva, and the cornea becomes hazy over the greater or less part of its surface. In from twenty- 
four to forty-eight hours the burnt surfaces are clearly mapped out, and are separated from the other tissues by a well-defined zone, in which the inflammatory reaction, the result of the injury, is most acute. Pus begins to be discharged, and the tissues destroyed by the burn are separated as sloughs, after which, if the injury to the eye has not been too great, recovery more or less complete commences.

Should the case, however, be severe, the inflammatory process goes on, the conjunctiva becomes thickened and fleshy, pus forms in the anterior chamber, the cornea suppurates and bursts, and the eye ultimately shrivels. In cases where the cornea does not give way, and the shape of the eyeball is preserved, a dense leucoma remains, or, from the thinning of the parts due to the separation of the sloughs, the cornea, unable to resist the intra-ocular tension, bulges forward, and a staphylomatous projection is produced.

Sequelæ.-In all severe burns both ocular and palpebral mucous membranes are destroyed, and the raw surfaces that are left adhere in the process of healing, so that the eyelid becomes firmly fixed to the eyeball. This condition, which is known as symblepharon, will, no matter how much care has been taken to prevent it, certainly occur in every case where the retrotarsal fold of conjunctiva has been destroyed; and, if it be extensive, it not only interferes with vision but causes intolerable discomfort fromthe sense of dragging which it produces. It is, unfortunately, but little amenable to treatment, and may be complicated by union of the eyelids-anchyloblepharon. 
Prognosis.-After a burn the healing process is slow. The vitality of the tissues has been lowered by the injury, and, as the cicatrix goes on contracting, the eyelids may either become inverted, so that the eyelashes rub upon the eyeball, or else they may be everted, with the result that the face is frightfully disfigured and the original damage to the cornea is augmented by the constant exposure of the ball.

Treatment.-Burns of the globe are treated in the same way as acute suppurative inflammation, and where the injuries have been caused by chemical irritants, special precautions have to be taken from the very first. In all cases, after the eye has been washed, castor oil and two per cent cocaine, or preferably a five per cent solution of chloretone in oil, should be applied to the conjunctiva, an iced compress laid over the closed eyelids, and the patient put to bed. In order to combat the inflammatory reaction which is sure to follow, the ice-cold compress should be continued, and saline purgatives administered, while the pain must be subdued by the application of leeches and either by the administration of full doses of opium or by hypodermic injection of morphia in the temple. The use of these anodynes has the additional advantage of inducing sleep and soothing the nervous system generally. After forty-eight hours, warm applications are usually found to be more grateful to the patient than the iced compress, and the sedative effect of these fomentations may be increased by poppy-heads and camomile flowers, while much additional relief may be obtained by smearing the eyelids, forehead, and 
temples with a mixture of extract of belladonna and glycerine. When the acute symptoms have subsided, and the sloughs have begun to separate, antiseptic lotions should be used, and much benefit is often obtained from the daily instillation of a two per cent solution of nitrate of silver, or of a two per cent solution of picric acid. Any iritic complications must be treated by the judicious use of atropine, but in this connexion it may be mentioned that as burns of the corneo-scleral margin are apt to give rise to glaucomatous symptoms, the use of the atropine must be at once stopped if the patient complain of increased pain with sudden dimness of vision, and an endeavour must be made to reduce the increased tension of the eyeball by the energetic use of eserine or pilocarpine, or by paracentesis of the aqueous chamber.

During the cicatricial stage the greatest attention must be devoted to overcoming the tendency to symblepharon. The adhesions must be diligently broken down with a probe, and the eyelids drawn away from the eyeball, so as to permit a layer of vaseline to be instilled between the two raw surfaces. In spite of all one's efforts, however, symblepharon will, in bad cases, occur, and although much ingenuity has been displayed in devising mechanical apparatus to keep the lid separated from the eyeball, these as a rule fail to accomplish their purpose, because, as cicatrization progresses, they get displaced, and the adhesions form again and again with a pertinacity that is most vexatious. 


\section{Explosion Injuries}

Signs and Symptoms.-These form a class by themselves. The skin is burnt and impregnated with grains of explosive and sand. The lids are usually so much swollen that they cannot be opened voluntarily, and are separated only with difficulty by retractors. The surface of the cornea and sclerotic is, like the skin, bespattered with grains, and there is always more or less subconjunctival ecchymosis. There is great pain, accompanied by intolerance of light, and lachrymation ; and in serious cases the lens is injured through foreign bodies having penetrated into the interior of the globe. There may be rupture of the cornea and sclerotic, and in all cases the eye suffers severely from shock. Superficial lesions gradually clear up, but sight is often more or less permanently impaired from opacities of the cornea. Whenever the globe has been perforated, the foreign bodies which have been driven into the eye set up iridocyclitis, which may go on to panophthalmitis.

Prognosis.-Obviously under such circumstances there is no hope of saving the eye, and after injury from a high explosive the prognosis ought always to be very guarded, because, even although there may not be much apparent physical damage to the globe, the degree and effect of the shock cannot at first be fully appreciated.

Treatment.-The treatment consists in bathing the eyes thoroughly with hot sterilized water, removing as far as possible all foreign particles, and instilling into 


\section{BURNS AND EXPLOSION INJURIES 5I}

the conjunctival sac castor oil in which the alkaloids of atropine and cocaine are dissolved in the proportion of one per cent of the former and two per cent of the latter. The patient ought to be put to bed as soon as possible, antiseptic fomentations applied to the eyes and face, a smart calomel purge administered, and anodynes given, if necessary, to relieve pain and induce sleep. After inflammation has completely subsided, it may be expedient, should vision be impaired owing to corneal opacities, to make an artificial pupil and to tattoo the scars, while at other times a cataractous lens may require to be extracted. Should there be a large lacerated wound of the cornea and sclerotic, immediate enucleation is advisable. 


\section{CHAPTER VI}

\section{SYMPATHETIC OPHTHALMIA}

Pathology.-The true nature of sympathetic ophthalmia, the most dangerous disease to which the eye is exposed, is still somewhat obscure; but recent investigations have shown that it is due to micro-organisms and their toxins travelling from the injured eye. It is usual to speak of the latter as the 'exciter', while the one secondarily affected is called the 'sympathizer'. The morbid changes in the exciter which are likely to give rise to sympathetic inflammation may be enumerated under the following heads :

I. Penetrating wounds of the ciliary region, accompanied by prolapse of the iris and ciliary body, are, above all others, the most likely to cause sympathetic disturbance, and all the more readily should the wound be lacerated and should the instrument with which it was inflicted have been unclean.

2. Foreign bodies lodged within the eyeball, more particularly if they lie near the ciliary processes, are a constant source of danger, since they keep up inflammatory reaction in the whole uveal tract.

3. Degenerative changes in an eye previously injured are always accompanied by a certain amount of iridocyclitis, and consequently an atrophied globe, tender and irritable through calcification of the lens 
and ossification of the choroid, is invariably a menace to the sound eye.

4. Corneal ulcers which have perforated may form the starting-point for a sympathetic ophthalmitis, but it is interesting to remember that an eye which has burst from within is not nearly so dangerous as one in which perforation has occurred from without. Moreover, all clinical experience goes to prove that after panophthalmitis the danger of a transference of infection from one side to the other is very slight.

The kind of eye which most frequently endangers the safety of the other is one which, in consequence of perforation through injury or ulceration, has been attacked by plastic iridocyclitis, and has become soft and tender to touch. The length of time which may intervene between the injury and the onset of sympathetic inflammation is very variable. It is very rarely less than three weeks, while, if the second eye escape until the one injured has healed, it will probably escape altogether, unless there be fresh inflammation or degenerative changes in the exciting eye. In the latter case the attack may not take place for many years.

Course.-The insidious nature of the onset is one of its most outstanding characteristics, for, although it is occasionally ushered in by severe pain and other symptoms of acute inflammation, it usually develops in a manner so treacherous that serious results are not anticipated until the disease is thoroughly established. It may make its appearance at any age, but is more frequent in the young than in the old, and particularly in 
those who are naturally delicate and of a neurotic temperament.

When once it has started it is extremely difficult to arrest. Even under the most favourable conditions it runs a protracted course, and, though recovery occasionally takes place, the result is very often total blindness. The destruction of the sympathizing eye is, indeed, often more complete than that of the one which received the injury, and the knowledge of this fact adds greatly to the responsibilities of the surgeon when he is called upon to treat a case in which sympathetic inflammation has fully developed and where the exciting eye still retains a fair amount of sight.

It is always necessary to draw a distinction between sympathetic irritation and sympathetic inflammation; for the former is simply a neurosis and passes off without leaving any organic changes, but the latter is plastic and in the long run involves the whole of the internal structures of the ball.

\section{Sympathetic Irritation}

Symptoms.-Sympathetic irritation is usually an early symptom. The patient feels that his eye gets soon tired; he has difficulty in reading small print; and after prolonged work he suffers from transitory attacks of dimness of vision when he looks at distant objects, or it may be from momentary total blindness. He feels uncomfortable in a bright light, which may cause neuralgic pains to dart through his head, and induce 
injection of the conjunctiva accompanied by copious lachrymation.

Signs.-If the field of vision be at this stage carefully examined by Bjerrum's screen the blind spot in the sympathizing eye will in many instances be found to have assumed a spindle-shape, and though I do not wish to insist too strongly on the value of this objective sign in the diagnosis of impending sympathetic mischief, I think it highly probable that, where there is an infected wound or degenerative changes in the other eye, it denotes active congestion of the optic disk, which may be regarded as a danger signal indicating the approach of genuine sympathetic disturbance. At this stage too the patient is sometimes found to suffer from a low degree of myopia, and the coexistence of both signs greatly strengthens the diagnosis. This temporary myopia may be due to spasm, but it sometimes persists under atropine, and then it can be explained only by supposing that congestion of the choroid has brought about an altered state of the media, whereby the refractive index has been increased. Whatever be the true significance of these signs, this much is certain, that both disappear after the removal of the exciting eye. For a time after enucleation the blind spot may persist in the form of a spindle, and so long as this is the case the patient continues to complain that his vision is weak and uncertain and that everything looked at appears 'wavy' and ' unsteady', but all these symptoms pass away as soon as the blind spot assumesits normal shape. Associated with the indications just noted ophthal- 
moscopic examination commonly reveals congestion of the optic disk, and increased fullness and tortuosity of the retinal blood-vessels. It is, however, always a difficult matter, when there is no healthy fundus for comparison, to determine by the ophthalmoscope alone whether the optic disk is, or is not congested: hence the value of the confirmatory evidence afforded by the coexistence of all these signs.

\section{Sympathetic Inflammation}

Symptoms and Signs.-Sympathetic inflammation may or may not be preceded by the symptoms just described; usually failing sight is the first warning that the patient receives of the development of the disease. When the eye is examined, a zone of pink hair-like vessels is seen surrounding the cornea; the iris is dull; and the pupil is small and sluggish, and dilates irregularly after the instillation of atropine.

Even at this early stage there may be neuro-retinitis and floating bodies in the vitreous, but more frequently the details of the fundus are obscured by haziness of the media incidental to inflammation of the uveal tract.

The cornea also becomes inflamed; spots form on its posterior surface-keratitis punctata; the aqueous is turbid, and the anterior chamber deep. The corneal signs are all the more marked when the inflammation assumes the serous type-serous iridocyclitis, but the plastic form is by far the more frequent and the more serious.

Blood-vessels now develop upon the surface of 
the iris, whose substance thickens and bulges into the anterior chamber, the exudation filling up the pupil, and later on gluing the whole posterior surface of the iris to the capsule of the lens (complete posterior synechia), matting the ciliary processes together, and implicating the choroid so extensively that the nutrition of the eye is gravely affected. As a result, the iris is retracted at its ciliary attachment, the tension diminishes, the lens becomes cataractous, and the shrinking vitreous causes detachment of the retina and of the anterior portion of the choroid.

Up to this time there has been perception of light, but now blindness becomes total. The eye is liable to recurrent attacks of iridocyclitis; its blood-vessels degenerate, and, rupturing, give rise to intra-ocular hæmorrhage ; it steadily shrivels, and the final result is phthisis bulbi.

Prophylaxis.-Since after sympathetic inflammation has fairly begun practically nothing can be done to check its progress, or to repair the damage which it has caused, the most effective treatment must be prophylactic, and we know that in the stage of irritation, and even in the early stage of serous iridocyclitis in the sympathizer, the removal of the exciter is followed by most beneficial results. Much of necessity depends upon the manner in which the injured eye has been treated immediately after the accident, and in the section on penetrating injuries of the eyeball a full description has been given of how eyes seriously injured may be saved and sympathetic inflammation prevented. 
Treatment.-In many cases, however, enucleation is inevitable, and the following rules may serve as a guide :

I. Enucleate at once when the injury is so severe that the exciting eye is destroyed hopelessly from the beginning.

2. Enucleate at once on the slightest sign of sympathetic irritation should the vision of the exciting eye only equal a perception of light and darkness.

3. Enucleate at once if the exciting eye is known to contain a foreign body and continues to be irritable.

4. Enucleate at once when an injured eye is blind and suffering from recurrent attacks of acute inflammation, or when it is tender and irritable as a result of the onset of degenerative changes-e.g. ossification of the choroid.

5. Do not enucleate when there is still sight in the injured eye, and when there is no sign of sympathetic disturbance in its fellow.

6. Do not enucleate when sympathetic inflammation is in progress, and there is still sight in the injured eye, for under these circumstances the removal of the exciter will have no beneficial influence, and the probability is that in the end all the sight the patient will possess will be in the primarily injured eye.

Optico-ciliary neurotomy, simple evisceration, and, as suggested by Mules, evisceration and the implantation of a glass ball within the sclerotic, have all been proposed as substitutes for enucleation ; but enuclea- 
tion is, undoubtedly, the safest operation from the point of view of prophylaxis.

In the treatment of the sympathetically affected eye, operation ought not to be attempted until all acute symptoms have subsided, for any attempt to perform a sclerotomy, or to excise a piece of the iris, even if successful at the time, is followed by an accentuation of the symptoms, and by increased outpouring of inflammatory exudation.

At the outset we must rely on the treatment suited to cases of chronic iridocyclitis. The eyes must be kept at rest, and this is best accomplished byshading them from the light and instilling atropine. If the inflammation be accompanied by great intolerance of light, lachrymation, and tenderness on pressure over the ciliary region, repeated leeching is useful in the early stages, but, in the latter, blisters to the temple or behind the ear are of more value.

Turkish baths are always helpful, but the number and frequency of these must be regulated by the patient's ability to stand them. Large doses of salicylate of soda are strongly recommended by Gifford and others, but here again great care must be taken that they do not depress the patient unduly. Owing to the recent discovery of certain changes in the blood in some cases of sympathetic ophthalmia, intravenous injections of salvarsan and neosalvarsan have been recommended, and reports of successful cases have been published. The treatment, however, is still on its trial, and more extended experience of it is necessary before any 
dogmatic statements can be made regarding its efficacy.

Pain ought to be relieved by the local application of heat, and by the judicious use of opiates, aspirin, or other sedatives. In all cases mercury is the one drug to rely upon, and this must be given until its physiological effects become apparent. It may be administered by the mouth, by subcutaneous or sub-conjunctival injections, by the calomel vapour bath, or by inunction. Of all these the last is probably the best, and a half to two drachms of mercury-vasogen should be rubbed well into the skin of the temple and brow every night. The action of the mercury is intensified by iodide of potassium, which may be given in ten- or twentygrain doses once a day after a meal.

It is very important, however, during this severe antiphlogistic treatment to attend carefully to the patient's general health. He must be well fed, and the appetite promoted by tonics, especially those containing quinine and iron. In a few cases recovery of sight may take place as a result of this treatment, but as a rule the most that can be hoped for is that the active symptoms will subside, leaving the iris firmly adherent to the capsule of the lens, which in all probability is now cataractous.

After a sufficient time- a year or eighteen months -has elapsed, and if, during that interval, there has been no recurrence of inflammation, the intra-ocular tension has not diminished, and the patient's perception of light be satisfactory, operative interference 
may be considered. If it be resolved on, the iris should as far as possible be left alone. The lens must be got rid of, either by needling, or, in more favourable cases, by drawing it off with a curette after the toughened capsule has been divided with a knife. After the cataract has been removed, an iridotomy may open up the pupil sufficiently to allow light to enter the eye and enable the patient to see as well as the damaged state of the retina will permit. It must always be remembered in operating on such eyes, that the vitreous is quite fluid and escapes readily, therefore it is important that all incisions be as small as possible and made wholly in the cornea.

After operation, the eye must be carefully bandaged, and the patient kept quiet in bed in a darkened room; while the local application of ice, and the administration of sedatives internally, will do much to prevent the occurrence of inflammatory reaction. 


\section{CHAPTER VII}

\section{AMBLYOPIA DUE TO TRAUMATIC NEUROSIS}

THE present war has made us acquainted with a large and interesting group of cases in which a man suddenly loses sight after having been exposed to shell-fire. The patients for the most part are nervous, excitable, and emotional; they are depressed and burst into tears on the slightest provocation; they all give a somewhat similar history of the onset of blindness, and in none is there any demonstrable organic lesion in the eyes themselves.

Symptoms.-The story given is usually that after a prolonged period of fatigue, or subsequent to the excitement and the nervous strain incident to heavy and continuous artillery fire, the patient finds himself suddenly incapacitated by the bursting of a shell. He has been thrown on the ground and, it may be, partially buried as a result of the force of the explosion, but in many instances he has received no detectable physical injury whatsoever. When found and extricated, if need be, from his unpleasant surroundings he is usually in a dazed condition, and although he may be able to walk, yet he has afterwards little or no recollection of any of the incidents accompanying this stage of his illness. He complains that he cannot see, and in the worst cases he is also un- 
able to speak, is deaf, and has lost the sense of smell.

At first the blindness is said to be absolute, but even then the external appearance of the eyes is quite natural, the pupils, though sluggish and occasionally unequal, react to light, and nothing abnormal can be detected on ophthalmoscopic examination. The patient may, however, complain that the light hurts him, and may suffer from blepharospasm whenever any attempt is made to examine his eyes.

Progress. - Sight returns gradually but much more slowly than hearing, smell, or speech, and one eye may recover completely while the other remains amblyopic. At first there is an intermittent glimmering of light, and complaint may be made of peculiar colour sensations. Afterwards large objects are seen, and, when vision returns sufficiently to permit the visual field to be measured, it is found to be greatly contracted. In spite of that, however, the patient at this stage usually guides himself about with little difficulty, and his behaviour with reference to his sight is strongly suggestive of what occurs in the amblyopia accompanying hysteria.

Treatment.-That gives the key-note to treatment, for these patients are in no sense of the word malingerers. The burden and the heat of the day have been more than their nerves could stand and they have broken down under the strain, but rest, good feeding, and, best of all, judicious encouragement help them at length to make a complete recovery. 


\section{CHAPTER VIII}

\section{OCULAR SIGNS AND SYMPTOMS ACCOM- PANYING HEAD INJURIES}

IN trench warfare injuries to the head are of very frequent occurrence, and papillœdema of the optic nerve often accompanies a depressed fracture of the vault of the skull due to a bullet or shrapnel wound. It is due to pressure, and disappears rapidly after a decompression operation. According to Colonel Lister, it occurs in fifty per cent of the cases and is the direct result of increased intracranial pressure, which he says may be caused by '(I) Swelling of the brain due to concussion, (2) Swelling of the brain due to inflammation, (3) Hæmorrhage on the surface of the cortex, (4) Abscess, or (5) possibly serous exudation from the meninges'. The eyes ought to be examined with the ophthalmoscope regularly at short intervals, for any increase in the papillœdema is a sure sign of increasing intracranial pressure which ought at once to be relieved by operation.

Injury to the occipital lobes is often accompanied by hemianopsia, and Colonel Lister as a result of recent observations states that ' (I) When the lesion occurs over the occipital cortex of only one side the result is either hemianopsia or homonymous quadrantic defect on the opposite side, (2) When the 
bullet passes through both occipital lobes there is at first complete blindness, as one might expect, but in several cases this gradually clears centripetally after an interval varying in different cases from hours to days, giving rise to a more or less central scotoma with a full peripheral field. In others one may get either hemianopsia with partial homonymous quadrantic defect on the opposite side combined with a central scotoma, or a central scotoma with a morbid tendency to hemianopsia.' 



\section{INJURIES OF NOSE AND T.HROAT}

BY

$$
\text { J. DUNDAS GRANT, M.D., F.R.C.S. (Eng.) }
$$

LATE MAJOR R.A.M.C. (POST OFFICE RIFLES VOLUNTEERS); KING GEORGE HOSPITAL, LONDON ; LORD KNUTSFORD'S SPECIAL HOSPITAL FOR OFFICERS;

AND

\section{H. LAWSON WHALE, M.D. (Camb.), F.R.C.S. (Eng.)}

CAPTAIN R.A.M.C. (T.) ; FORMERLY NO. I3 GENERAL HOSPITAL, BRITISH EXPEDITIONARY FORCE, OVERSEAS; THE COUNTY OF LONDON.WAR HOSPITAL, EPSOM. 
In the preparation of the following chapters on Wounds of the Nose and Throat, much valuable information has been derived from

(a) Considérations Cliniques surl'Oto-Rhino-Laryngologie en Temps de Guerve (Professor Moure's Military Clinics), Bordeaux, I9I5, by Dr. Georges Canuyt.

(b) Traité de Chirurgie de Guerre, Paris, I878, by Dr. E. Delorme.

(c) Surgical Experiences in South Africa, by Sir G. H. Makins.

(d) A Civilian War Hospital, by Sir A. Bowlby and others.

(e) Über Kriegsverletzungen am Ohr, den oberen Luftwegen, \&c. (Archiv für Ohren-, Nasen- und Kehlkopfheilkunde, Feb. I9I5), by Prof. Denker.

(f) Drei Kriegsverletzungen des Kehlkopfes, and Weitere Erfahrungen über Kriegsverletzungen des Kehlkopfes (Zeitschrift für Ohrenheilkunde, \&c., January and March, I9I5), by Prof. Koerner.

We are indebted to Messrs. Churchill for kind permission to introduce Figures 4 and 7 taken from Hughes's Practical Anatomy.

J. D. G.

H. L. W. 


\section{WOUNDS OF THE NOSE}

In dealing with wounds of the nose and nasal cavities in warfare, we must bear in mind at the outset that their gravity differs according to the direction and the nature of the projectile.

Influence of Direction of Projectile.-A bullet directed transversely may simply penetrate and perforate the projecting portion of the nose, or even the maxillary antra, after the fashion of a setonas the French describe it-leaving little more than the traces of the wounds of entrance and exit respectively, with extraordinarily little damage. On the other hand, a wound in the antero-posterior direction is likely to involve the superior maxilla and the vertebral column, and an obliquely directed bullet is likely to damage some of the neighbouring organs, such as the skull, the brain, the orbit, the great vessels, nerves, and ears. As an exception to these general rules we find some remarkable instances of bullets -which, judging by their points of entrance and exit, must seemingly in their course have damaged important structures; and yet it is found that, as a fact, these structures have escaped injury; as exemplified in Cases 9, 20, 21, 22, and 23.

Influence of Nature of Projectile.-As a general rule, a rifle-bullet makes for itself a clean passage, doing relatively little damage, as in Cases 8, I3, 21, 22, 23, 
$3 \mathrm{r}$, and 34; whereas the round bullets of shrapnel tear and shatter, as in Cases 24, 26, 27, 28, 29, 30, and 33; while fragments of shell cause still more extensive laceration. It is true, however, that shrapnel, if spent, either comes to rest very soon, as in Case II, or it does not even penetrate, as in Cases 4, 5, 6, and ro. Again, a rifle-bullet, which has been reversed so as to travel base forwards, produces effects similar to those of shrapnel, as in Case I2. Such reversal of a bullet may occur either on impact or, according to some authorities, in the air before impact, at very long ranges. Finally, there are incontrovertible cases in which the bullet has been deliberately reversed in the cartridge, before the loading of the rifle.

General Anatomical Considerations. - The nose may for our purpose be considered as consisting of three portions: the external nose, the nasal cavities, and the accessory cavities. A few of the salient features of each of these may be helpful to those who do not make a special study of the part.

As a whole, the nose is formed by the nasal fossæ separated from each other by the septum, each having on its outer walls the three turbinated bodies which occupy a considerable amount of the available space. These cavities are covered in in front by the external nose, which is bisected by the septum; and they open below at the two nostrils. The nasal fossæ have opening into them by small apertures the accessory cavities, namely, the antra of Highmore on each side, the frontal sinuses above 
and in front, the ethmoidal sinuses at the sides and above, and the sphenoidal sinuses posteriorly.

Surgical Anatomy.-For surgical purposes, the structure of the external nose is fairly obvious, and only a few points need to be recalled with regard to which the practitioner's memory may be somewhat hazy.

External Nose.-The prominence of the nose is partly bony and partly membrano-cartilaginous. The bony portion is formed mainly by the nasal bones proper and the frontal or ascending processes of the superior maxillæ. The nasal bones articulate by a deeply serrated synostosis with the notch in the frontal bone which overlies the articulating portion. In the bare skull the nasal bones project much more horizontally than a consideration of the normal average face would lead one to expect. On each side, the outer edge of the nasal bone articulates with the ascending process of the superior maxilla, which forms a considerable part of the side of the bony part of the external nose. The pair of nasal bones is thus wedged between these ascending processes, and in such a way that they rest upon them while being gripped between them. At their median line of junction the nasal bones present two ridges which, projecting backwards, together form a portion of the nasal septum and articulate with the anterior part of the perpendicular plate of the ethmoid, as also with the nasal spine of the frontal bone.

The ascending processes of the superior maxillæ 
form the side parts of the bony portion of the external nose which can be gripped between the finger and thumb, and felt to be rigid. Their anterior edges enter largely into the formation of the sides of the 'pear-shaped' orifice of the nose as seen in the bare skull. The direction of the lower part of the margin

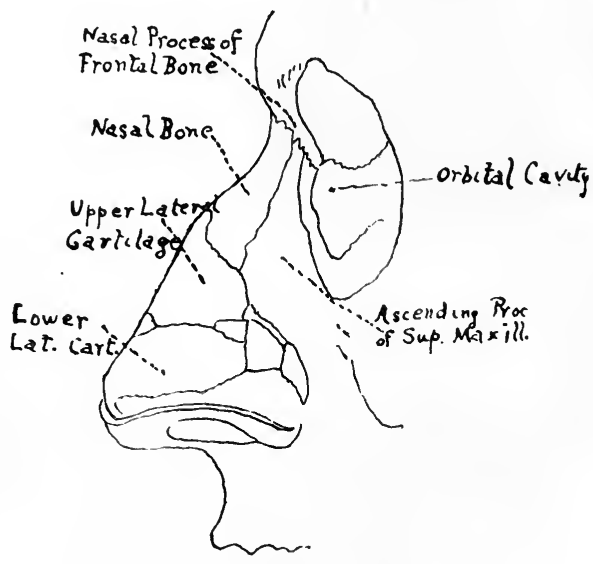

Fig. i. The Externai, Nose.

is backwards towards the canine tooth; it then curves forwards horizontally, parallel to the alveolar margin, here forming the floor of the pyriform fossa. The median portion of this opening presents below, at the junction of the two superior maxillæ, the nasal spine, which forms the strongest and lowest part of the nasal septum.

The sides and dorsum of the mobile portion of the external nose are formed mainly by the lateral 
cartilages, upper and lower, which are attached to the margins of the pyriform opening.

Nasal Fossæ.-The nasal fossæ proper, separated from each other by the septum, are narrower above than below and two inches in height. Their roof is formed mainly by the very delicate cribriform plate

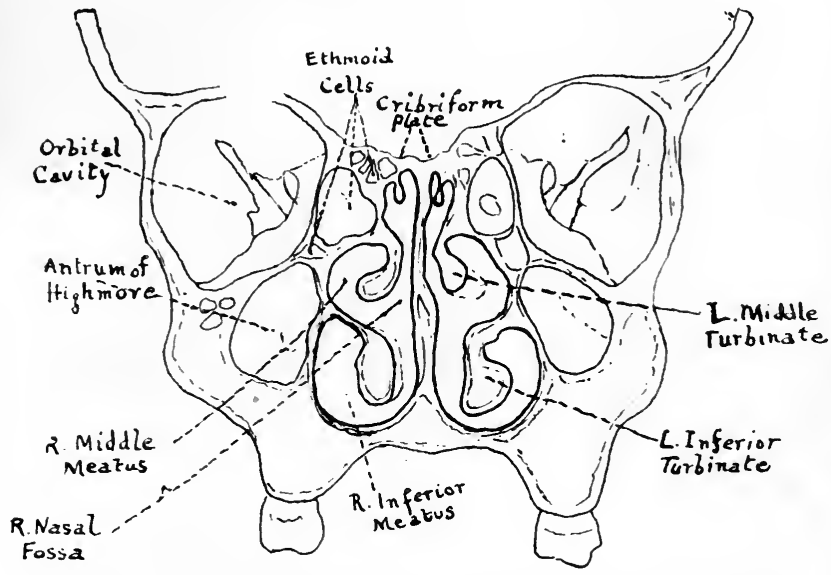

Fig. 2. Coronal Section through Nase Fosse and adjacent Cavities.

of the ethmoid, their floor by the palatal processes of the superior maxillary and palatal bones. On the outer walls are the three turbinated bodies which project as curling shelves into the cavities. Posteriorly the upper one third of the cavities are closed in by the body of the sphenoid bone, the lower two thirds opening into the naso-pharynx. 
The Nasal Septum.-The septum acts as a partition between the two cavities, and in relation to wounds and injuries presents points of considerable importance. Its osseous part consists mainly of the perpendicular plate of the ethmoid and the vomer. These diverge in front at an angle of about $37^{\circ}$, which

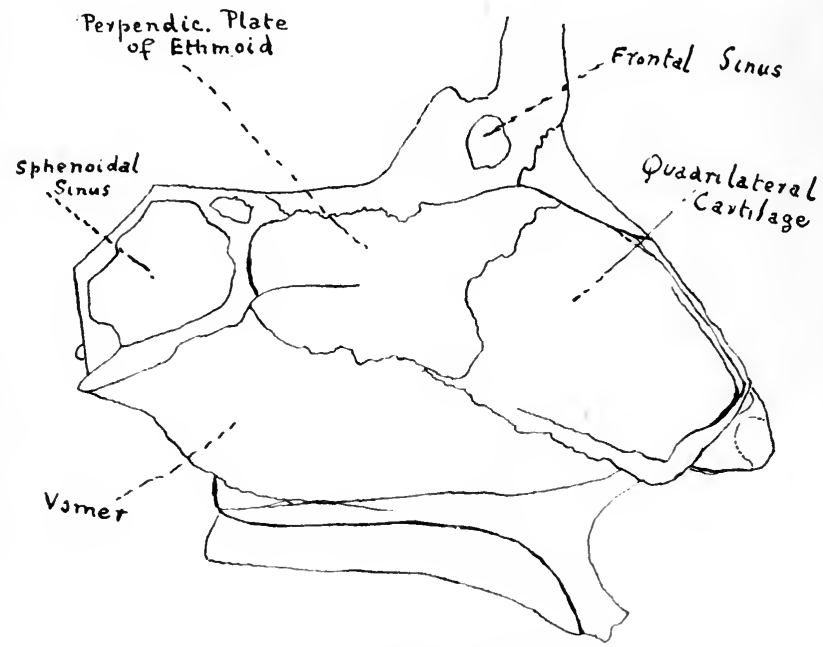

Fig. 3. The Nasal Septum.

is filled in by the posterior part of the 'quadrilateral septal cartilage'. This extends forwards into the external nose, and is attached antero-superiorly to the nasal bones, the upper and the lower lateral cartilages. The septal cartilage is frequently bent or broken by blows on the nose. Bending (as in Case I) causes nasal obstruction, and fracture is usually 
accompanied by a dislocation backwards and downwards from its attachment to the vomer. This is followed by traumatic hæmatoma of the septum and by a typical deformity-a depression between the nasal bones and the tip of the nose. A knowledge of these points is very important in the treatment of fractures of the nose.

The Accessory Sinuses.-The accessory cavities are the maxillary antra, the frontal sinuses, the ethmoidal cells, and the sphenoidal sinuses.

The maxillary antra occupy nearly the whole of the superior maxillæ on each side of the nasal fossæ, into which they open by small apertures hidden by the middle turbinated bodies. Pus in them runs into the nasal cavity when in sufficient quantity to overflow, or when the head is bent forwards and to the opposite side. Bullet wounds through the antrum usually fracture the orbital plate of the superior maxilla, as in Cases 7, 9, and 18.

The frontal sinuses, lying between the outer and inner plates of the frontal bone, are just above the orbits and may extend far back over the roofs of these cavities. They vary much in size, but are generally sufficiently large to hold a bullet. The flow of pus, when present, is greatest when the head is erect. For a fatal instance of a perforating bulletwound, see Case I9.

The sphenoidal cells occupy more or less asymmetrically the body of the sphenoid bone which lies above the naso-pharyn $x$ and closes posteriorly the upper one third of the posterior aspect of the nasal fossæ. 
On the roof of the sphenoidal cell we have the sella turcica with the pituitary body and the optic chiasma. On the outer side lie the optic nerves. The orifice of the sinus is small and is situated in front and high up. The lateral wall of the sinus is thin ; a consideration of the structures which lie in close contiguity will remind us that any infective wound of this sinus may have one or more of the followingsequelæ:-meningitis, cavernous sinus thrombosis, optic neuritis and blindness, and internal-or less often external-strabismus.

The ethmoidal cells encroach upon the upper half of the nasal cavities and extend outwards to the inner wall of the orbit, the partition being formed mainly by the os planum of the ethmoid, and to a less extent by the lacrimal bone in front and the orbital plate of the palate-bone and the sphenoid behind. They are roofed in chiefly by the horizontal portion of the frontal bone and part of the lesser wing of the sphenoid. The cribriform plate of the ethmoid does not actually form part of the roof of the ethmoidal cells, being placed mesial to these. But the cribriform plate here demands mention, because it is situated in such immediate contiguity to the ethmoidal cells that in any interference, accidental or deliberate, with the parts in the higher regions of the nasal fossæ, it is often damaged. The slightest injury to this plate is likely to result in meningitis or cerebral abscess, the infection passing either directly through a fissure in the very thin bone, or along the lymphatic sheaths of the branches of the olfactory nerve. Lastly, it is unusual for a bullet to penetrate the 
ethmoid without damaging some more vital part. An antero-posterior wound generally reaches the brain. A transverse wound usually traverses the ethmoid of both sides, and destroys one or both eyes. This close relation of the orbit to the ethmoidal cells is well shown in Fig. 2 and exemplified in Case I7.

Wounds of the External Nose.-These may take the form of slits, notches, or more or less complete detachment of parts. A nose has been known to be almost entirely detached, or even removed altogether. In treating such cases, it is important that no parts should be removed for the sake of trimming up, but they should be sutured in their place. No doubt this is hardly possible on the field of battle, but at the dressing-station it should be carried out, if possible. It is important that corresponding parts should be adapted one to another, and this is particularly the case in regard to the septum, in order to avoid subsequent falling in or detachment of this part of the nose. If direct suture of the septum is impossible, antiseptic tampons of smooth shape and equal thickness may be placed in each nostril, or, as a counsel of perfection, a small piece of rigid drainage-tube or gum-elastic catheter wrapped round with gauze may be inserted in each. In Case ${ }_{5}$ the septum was forcibly straightened.

Hæmorrhage at the time of the wound has to be stopped, as circumstances will best permit, by the pressure of the fingers or the soldier's first field dressing, or it may be necessary to insert pledgets of gauze. At the dressing-station or field-ambulance 
these may be removed and replaced. If an application of cocaine and adrenalin, either liquid or in the form of an ointment, can be made, this is desirable ; and in these, as in all nose wounds, cleansing may be effected by bathing with 5 volume peroxide of hydrogen and normal saline solution. In any case the surrounding skin should be painted with tincture of iodine.

Gunshot wounds may simply fracture the framework or may cause the nose to be chipped, notched, or completely perforated (see Case I). Much depends upon the direction, and the above results only occur when the bullet is directed transversely. It is more common, however, for wounds of the external nose to be complicated by damage to neighbouring parts, such as the spinal column or one or other of the maxillæ; or the bullet may lodge in the antrum or other nasal sinus, or in the palate, as in Cases II, I2, and I8. In dealing with these in the early stage it must be again remembered that no portions of the tissues should be removed which can possibly be preserved. The parts must be purified and hæmorrhage arrested, as before stated. Any absolutely loose chips of bone or foreign bodies may, however, be removed. An endeavour should be made to bring the parts together, but without interfering with drainage, and as a rule it is advisable to allow the inevitable swelling of the parts to subside. If the nasal bones or cartilages are depressed, they are to be lifted up by means of the finger or dressing-forceps introduced into the nostrils, but little can be done 
in the way of keeping them in position, as the presence of intra-nasal tampons is contra-indicated on account of sepsis. In the absence of swelling, the lateral displacements may be met by the application of a casing of zinc or plaster, but under war circumstances this in the earlier stage is practically out of the question.

Deformity.-A considerable amount of consecutive deformity is not uncommon and may take the form of flattening of the base of the nose, lateral deviation, or even complete collapse and considerable narrowing of the nostrils ; or, on the other hand, a large or small degree of laying open of the nasal fossæ. Such an opening may be surrounded with shreds of tissue folded irregularly on the margin of the hiatus. In some cases a lachrymal fistula is formed, and, as Makins observed in the South African War, the sense of smell may be either diminished or lost. When the nostrils are narrow, respiration is difficult, and the voice may be affected, or, from loss of the protecting materials of the nose, respiration through it may be unaccompanied by the normal degree of moistening and warming, so that the individual is exposed to frequent attacks of catarrh and bronchitis.

In these more extensive degrees of destruction it may be impossible to do anything for days or weeks except to cleanse the parts and endeavour to reduce the swelling. The rhinologist himself would have to stay his hand. Our resources are the frequent use of a warm 5 volume solution of peroxide of hydrogen, and the continued application of moist dressings, such as fomentations of boracic acid or of liq. alum. 


\section{8o . INJURIES OF NOSE AND THROAT}

acet., one part in eight of hot water. Hypertonic saline ( 5 per cent. with $\frac{1}{2}$ per cent. citrate of sodium) has a definite action in inducing local osmosis and so flushing the part with lymph. Clinically, its value for very septic, gaseous, or chronic wounds is becoming increasingly evident, since Sir Almroth Wright first advocated its use in war. It is used to irrigate cavities, or as a dressing on raw surfaces, or for moistening gauze plugs for wounds. The dressing may, with advantage, be covered with jaconet, oilsilk, or other impermeable material. The frequency with which it is to be changed necessarily varies with the degree of septicity.

By the time this is accomplished we arrive at a later stage, and various undesirable cicatricial changes may have taken place, the most preventable of which is adhesion between the turbinated bodies and the septum, as in Case 2; although in favourable cases-i. e. where sepsis is only slightly marked-the application of cocaine and adrenalin may help to keep down turgescence of the mucous membrane until healing of the damaged surfaces has taken place, nevertheless more often these adhesions cannot be avoided and have to be rectified afterwards. The best way to relieve them is removal of a portion of the turbinated body and any spurs or projection on the septum (Case 2). In many cases, however, the parts are so embedded in cicatricial tissue that this process cannot be carried out. Moure finds that he can then deal with this difficult condition by detaching the ala of the nose at the nasolabial furrow, by which he attains free access. He then 
scoops away the cicatricial tissue, introduces a tampon of iodoform gauze, and restitches the ala. He does this on one nostril at a time, leaving the second one until healing of the first has become well established.

Occasionally the outer nose is displaced by the injury and undergoes reunion in a faulty position, so that the nostrils are completely closed. Detachment of the ala, as practised by Moure, may make it possible to reimplant the nose in proper relation to the nasal passages. A case is recorded in which, although the nose had been put in good position immediately after the injury, it got displaced during transport and had to be replaced by operative measures.

A wound, severing the nose from the cheek, may pass backwards right through the antrum and the spheno-maxillary fossa, as in Case 32 .

From the nature of modern warfare, sabre-cuts are rarely met with.

Extreme disfigurement, in addition to much suffering and functional disturbance, is caused by extensive wounds of the external nose and superior maxilla. Large portions of the parts may be removed or the root of the nose may be driven in so that the nostrils are directed straight forwards and are usually occluded, while the mouth is laid open by the shattering of the jaw and tearing up of the lips and cheeks. Cases of varying grades of injury are Nos. 28, 30, 3I, 32 , and 33 .

In the present war, in which the fighting has been largely a matter of trench warfare, such wounds have

R.G.W. 
been of regrettable frequency. The slighter of these are inflicted by rifle-bullets, but the more severe by portions of bursting shells and perhaps the worst by hand grenades at close quarters. Here the principle of preserving with care all the bony and soft parts should be acted upon and an attempt made to coax them into their normal position (Cases 20 and 3I), endeavouring, if possible, to maintain the nasal passages. Of course, absolutely loose portions of bone may have to be removed, either because it is obvious that their reunion is impossible or because they are keeping up a purulent discharge. During the process of purification, adhesions inevitably follow, and if the case is left at this stage, the disfigurement may be extremely distressing.

Plastic Surgery.-To remedy this, the art of plastic surgery of the face comes to our aid, but at the same time the resources of nasal, maxillary, and facial prothesis are sometimes sufficiently satisfactory. Délorme, indeed, expresses a preference for nasal prothesis rather than rhinoplasty, in suitable cases.

If we propose to carry out rhinoplasty, the question arises as to the period at which it should be done. In view of the extraordinary septicity of such injuries in the present war, the prevailing opinion is that plastic treatment should not be undertaken until some time after recovery has taken place. Dr. Kisch considers that a fresh outbreak of the sepsis is liable to take place in the nose just as it does in other parts.

In the typical deformity of the nose described above, M. Morestin at the Val de Grace Hospital in 
Paris, performs, as a rule, an operation consisting in the detachment of the nose from the remains of the nasal bones, so as to turn down the projecting portion and the tip (as in Ollier's operation) and in due time the filling up of the gap by a flap from the forehead in which a portion of cartilage from the eighth rib has previously been implanted. The formation of an upper lip is generally achieved by detachment of the soft parts from the cicatricial adhesions to the subjacent bones, lateral undercutting incisions of relaxation, and the approximation of the remaining parts with as little trimming off as possible, towards the middle line.

A remarkably complete description of the various plastic operations of the nose is contributed by Joseph C. Beck to Loeb's Operative Surgery of the Nose, Throat and Ear (I9I4), also in the Surgery of Deformities of the Face, by J. B. Roberts (London, I9I2), and the details of restorative prothesis and the remarkable scope for the skill of the dental surgeon in replacing lost portions of the jaws, are given very fully in a work on the subject by Martinier and Lemerle (Baillière, Paris, I9I5). French dentists or stomatologists have thrown themselves into this work with peculiar ardour.

Cases which were treated in France with a view to the fitting of a prothesis at home are illustrated by Nos. 24 and 27. No. 25 shows the use of wiring; while in slighter cases, as in No. 29, neither a plastic operation, wiring, nor a prothesis is necessary.

Wounds of the Nasal Fossæ.-These involve the 
contents of the fossæ and in particular the inferior turbinated bodies which are often cut up, as in Case I, and are apt to form adhesions with the septum so as to produce obstructions. Could these be treated early on rhinological principles, such obstructions might be prevented, if at the same time the occurrence of sepsis could be guarded against.

In warfare, however, the avoidance of sepsis seems nearly impossible, and early expert rhinological skill is not yet generally available. Under these circumstances we have usually to deal with the cases after cicatricial contraction has taken place (Case 2).

In recent wounds of the nasal fossæ, before cicatrization, timely rhinological interference may prevent obstructive adhesions. Such measures as the application of cocaine and adrenalin or menthol may suffice to produce contraction of the turbinal mucous membrane.

When cicatrization has already taken place, the case has become more difficult. Obstructions of the nasal fossæ not merely interfere with respiration but they help to perpetuate suppuration and to favour infection of the accessory sinuses.

The method of dealing with such cicatricial conditions must depend on the details of the individual case.

In some we may have merely a limited synechia between a turbinal and the septum, to be treated on ordinary rhinological lines. If the adherent turbinal is much hypertrophied, the most expeditious and reliable method is the removal of the portion adherent to the 
septum, but so extensively as to be well clear of the adhesion. Should there be also a spur or other deviation of the septum, this may be dealt with after the turbinotomy wound has healed, for obvious reasons. If adhesions of a turbinate to soft parts has occurred, excision of part of the lining of the outer wall may be necessary.

Wounds of the Maxillary Sinus.-The gravity of these varies according to whether they are gunshot wounds or shrapnel wounds, and whether the projectile has lodged in the cavity or has passed through it.

A bullet directed transversely may perforate one or both antra without leaving any particular trace, as in Cases 2, 8, and 13. More often, however, suppuration follows. One of us has found this inevitable if, in a bullet wound, the bullet lodges and remains in the sinus (Case I2); and in all shrapnel wounds, whether the shrapnel remains in the sinus or not (Cases 7, 9, Io and I4). Evidence of lodgement has often been afforded by X-ray examination, and this is almost a matter of routine. X-ray pictures of the nasal cavities and accessory sinuses are difficult to produce, and difficult to interpret. The bullet must be localized in all three dimensions of space; and Cases I4 and 18 illustrate the familiar truth that reliance cannot be placed on one picture taken in one plane only. Wound-probing is used much less frequently than formerly, in view of the information obtainable by X-ray examination, transillumination, and other rhinological methods. 
For the removal of a bullet or fragment of projectile from the antrum, the cavity should be opened through the canine fossa, as described below. This is as a rule easy to carry out, but it may be rendered more difficult than usual by the presence of cicatricial adhesions and thickenings, displacements of osseous parts or other direct or indirect results of the traumatism.

After the removal of the foreign body, the gentle curetting of the sinus, and the formation of an opening for free drainage into the nose, the suppuration usually subsides with rapidity, fistulæ heal, and general improvement takes place.

The opening of the sinus is a frequent occurrence in the practice of the rhinologist, and many refinements have been devised for the obtaining of the best results in cases of disease, but the following simple method is applicable to most of the cases met with as the result of warfare.

Operation on Antrum.-The upper lip being raised, an incision may be made above the alveolar process from the canine to the first molar. The periosteum should then be divided, and with an elevator should be raised until the canine fossa is freely exposed. With a chisel a small opening may be made, and a large round burr may be employed to enlarge it to such an extent that the little finger may be introduced. In this method of opening, the largest aperture is made with the least removal of bone, and the least disturbance of such teeth as may still be in situ. Some form of forceps can then generally be introduced for the removal of the bullet or other foreign body, and 
of course, if necessary, the opening can be enlarged by means of a gouge and mallet, or preferably a small punch forceps. It is then desirable to make an opening through the inner wall of the antrum into the nose. The interior of the antrum should be scraped and disinfected with chloride of zinc $\left(\frac{1}{2} \mathrm{dr}\right.$. to I oz.) ; an opening should be made through the inner wall into the nose, through which the end of a strip of gauze may be passed and guided to the external naris, while the continuation of the strip is packed into the antrum itself. The incision over the alveolar process may be stitched and allowed to close up, but it is very convenient to leave it open, so that after the iodoform gauze has been withdrawn the patient can at any time take a mouthful of some antiseptic liquid, such as boracic acid or a solution of hydrogen peroxide, and with a movement of the buccinator, force it through the antrum and out by the nose. Very little food ever gets into the antrum, in spite of the presence of the opening, and the irrigation as described should be carried out after each meal.

Further refinements in the details of the operation are found in all handbooks of nasal surgery.

By this route, known as the Caldwell-Luc, shrapnel was removed in Cases 9 and I4, and in several other antral cases, in which no shrapnel was found, comminuted bone was removed by this means of approach.

Those who might advisedly hesitate in dealing with any of the other accessory sinuses, may with all confidence attack the antrum of Highmore, knowing that 
the risks are comparatively small and the prospect of benefit enormous.

Experience seems to show that these injuries of the sinuses are extremely amenable to treatment and very satisfactory in their results, more especially in comparison with those of the nasal cavities proper. In the latter, the cicatrization often produces conditions which can only be dealt with with great difficulty and only moderate success.

It is, therefore, of the utmost importance that they should receive special care as early as possible, before faulty adhesions have taken place.

Sequelæ of Sinus Wounds. - We must not leave the subject of wounds of the accessory nasal sinuses without a reference to complications and sequelæ of infections of any of them. Thus, the continued presence of pus will eventually ruin the patient's general health; its repeated inhalation may lead to laryngitis, septic pneumonia, or bronchiectasis; and from being constantly swallowed it may entirely derange digestion. These happenings are among the more remote, which will probably come under the notice of the patient's medical adviser some time later, and at home. There are, however, other and very immediate possibilities which the surgeon must from the outset bear in mind. Infection may extend to the orbit, or to any of the structures behind the orbit in the sphenoidal fissure; among these grave sequelæ the most hopeless is cavernous sinus thrombosis. The other accessory sinuses of the nose may be infected in sequence. An infection by various 
routes, often the cribiform plate, may reach the anterior fossa, producing meningitis or cerebral abscess. A fulminating attack of erysipelas or cellulitis may indicate that the infection has spread to the overlying soft tissues. Sometimes, despite adequate drainage, copious pus continues to be formed, until rigors herald the onset of a spreading osteo-myelitis, which, especially in the case of a frontal sinus, the most careful and vigorous measures may be powerless to check. The possibility of septicæmia after sinus injuries must not be forgotten. . Lastly, it is no uncommon occurrence for an infection of the nasal cavities or accessory sinuses to spread up the Eustachian tube to the middle ear, resulting in acute suppurative otitis media.

Tetanus may occur, just as in other parts of the body.

This subject is fully dealt with in the section of the primer devoted to general surgery; as are also the details of diagnosis of the various local and constitutional complications above outlined. But among the more significant indications of danger, present or threatening, for which the surgeon should be at all times on the alert, we would emphasize the following : pain in any part of the head, including the ear; mental apathy; changes in the countenance, such as suffusion, loss of normal creases or expression, asymmetry ; ocular disturbances, for instance squint, unusual size or inequality of pupils, lack of purpose in direction of vision, sub-conjunctival hæmorrhages ; epistaxis; and systemic upsets, as indicated by the temperature chart, rigors, and the appearance of the tongue. 


\section{WOUNDS OF THE THROAT}

Anatomy.-The throat may be considered to comprise the pharynx, larynx, the upper parts of the trachea and œsophagus, and the antero-lateral region of the neck.

This antero-lateral region has, as its upper limit, the lower borders of the body of the inferior maxilla, and the line from here to the mastoid apex, and as its lower limit the episternal notch and the superior borders of the clavicle and acromion process.

The region is divided up by systematic anatomists into various spaces or triangles, and for localization purposes it is advisable to consider the limitations of these spaces, with the structures they contain.

For practical purposes, however, we may adopt Delorme's simpler method of division, giving us the following regions :

Regions.-The supra-hyoid, which includes the sub-maxillary triangles and the upper half of the superior carotid triangle.

The sub-hyoid, bounded by the hyoid bone, the episternal notch and the anterior borders of the sterno-mastoids. This includes what anatomists call the inferior carotid triangles and the lower half of the superior.

The sterno-mastoid region, or part covered by the sterno-mastoid muscles. 
The supra-clavicular region in a wider sense, corresponding to the 'posterior triangle' of our anatomists.

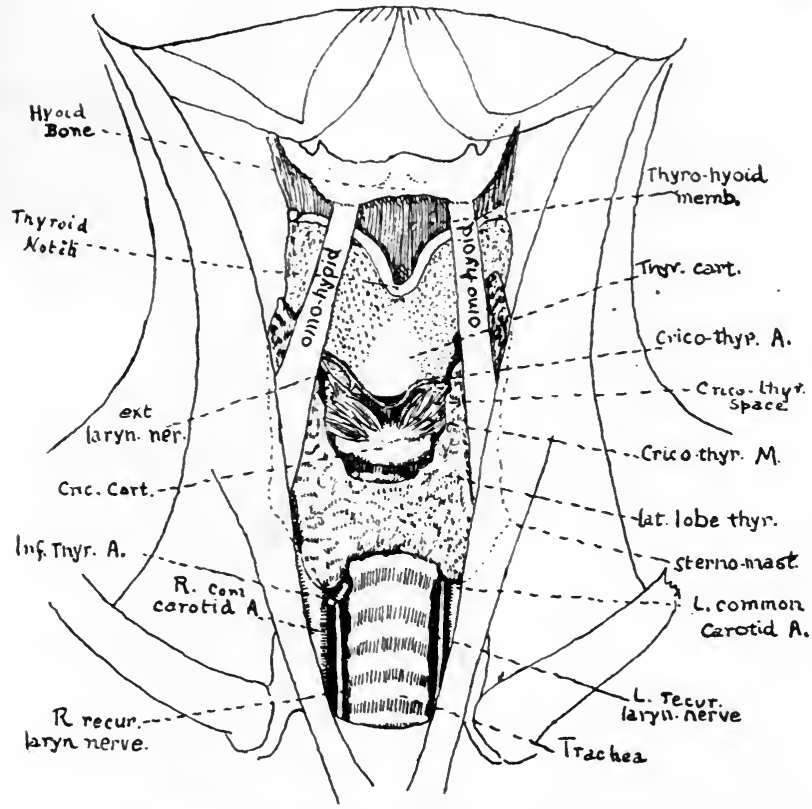

Fig. 4. Main Structures in the Front of Neck.

Parts endangered in various regions.-Wounds in the supra-hyoid region may, therefore, involve the sub-maxillary glands, the common facial vein, the hypoglossal nerve, the superior laryngeal nerves, 
and more deeply the base of the tongue, the epiglottis, the pharynx, and lower down the hyoid bone.

In the sub-hyoid region the parts liable to be involved are the thyroid body, the larynx, the

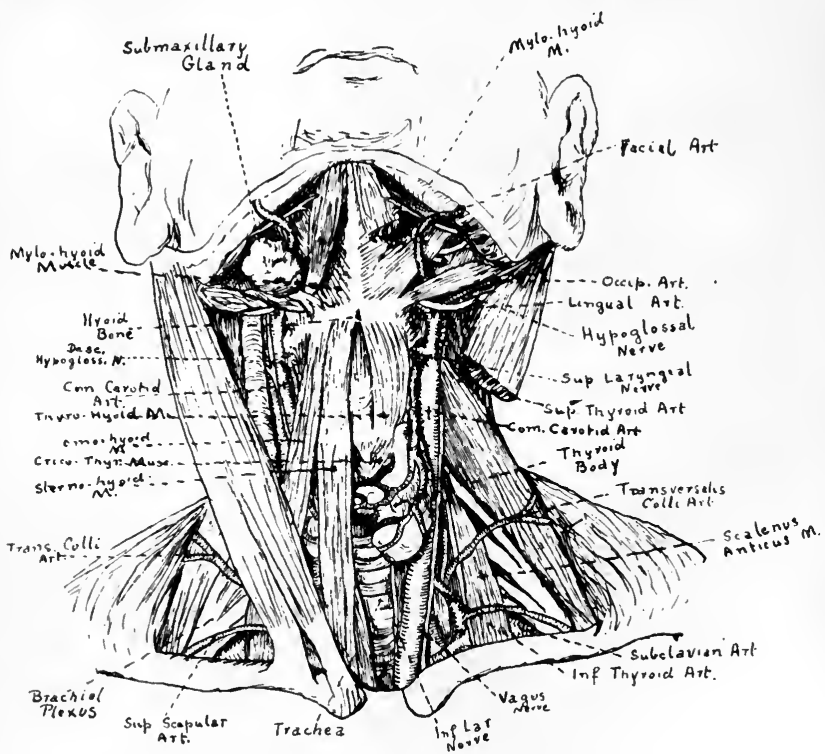

Fig. 5. Main Structures in the Front of Neck, SHOWING THE DEEPER PARTS ON LEFT SIDE.

trachea, the œsophagus, the superior and inferior thyroid arteries, the thyroid veins, and the superior and inferior laryngeal nerves.

Wounds involving the parts covered by the sternomastoid muscle endanger the carotid vessels and their branches, the accompanying veins and nerves. 
In the supra-clavicular region, wounds are apt to involve the sub-clavian vessels and branches, the

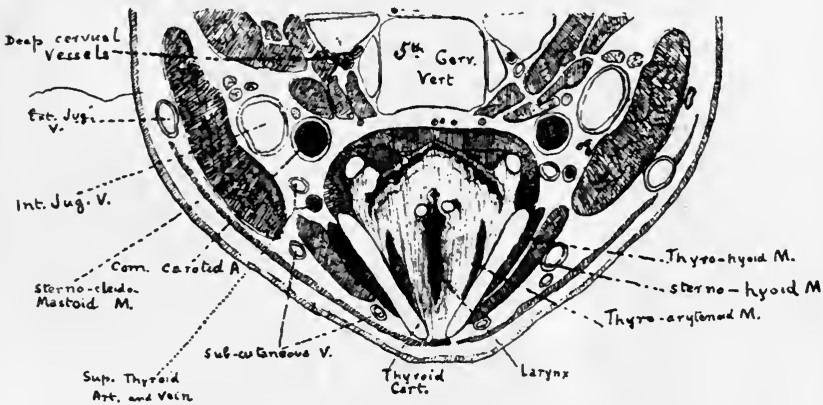

Fig. 6. Transverse Section of Neck at level of the UPPER Part OF LARYNX.

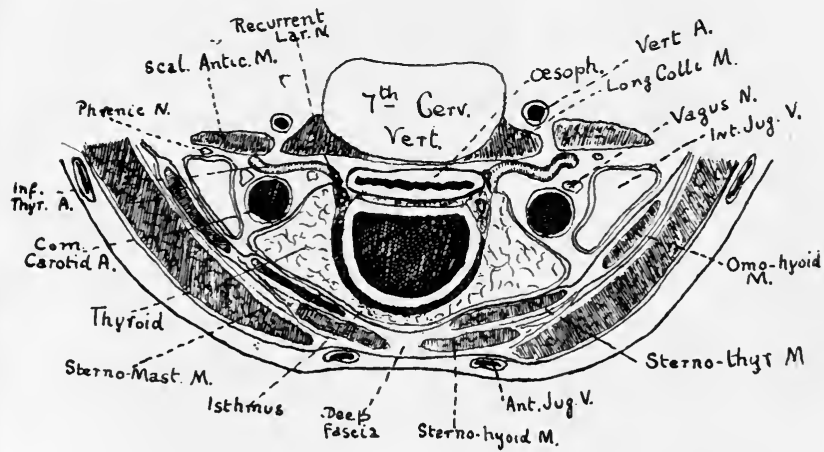

Fig. 7. Transverse Section through Lower Part of NECK.

external jugular vein, the roots of the brachial plexus, the great lymphatic channels and even the cervical dome of the pleura and apex of the lung. 
Venous Hindrances.-Surgical interference in one or other subdivision of the neck is frequently necessary in war. The parts of the neck where an intricate anatomical relation of important structures makes such interference specially formidable, are the carotid and the supra-clavicular sub-divisions of our anatomists. The practical point is, that in each of these, access to deeper structures is hindered by large veins, which are often plexiform, and demand much more respect on the operating table than in the dissecting-room.

Thus, in the carotid triangle, the lingual vein may join with the common facial, which again may communicate with the superior thyroid, and from the common facial, just as it disappears under the sterno-mastoid to empty into the internal jugular, a large branch passes downwards and slightly forwards, eventually to join the anterior jugular.

Similarly, in the supra-clavicular triangle, the supra-scapular and transversalis colli veins often form a plexus so dense as to be a veritable barrier to the sub-clavian artery. If, in addition to this, the field is obscured by hæmorrhage, it may even be necessary to resect part of the clavicle before tying the main trunk.

Selection of Route of Access.-When the necessity arises to explore a wound or expose a deep structure in the neck, it is not always at first glance obvious whether the anterior or the posterior triangle allows of the quickest and safest dissection. Of course, in a wound, for instance, of the thyroid cartilage, the 
anterior, and for a bullet lodged on the transverse process of a cervical vertebra the posterior, triangle would be elected. But there are cases in which, even with the aid of a good skiagram, it is impossible to determine the relation of the foreign body to the deeper structures of the neck, and in particular, to the wind-pipe and gullet; sometimes there is definite evidence that both these have been damaged. In any doubtful cases we would advise the surgeon, in an emergency, to cut down in the posterior triangle. The anatomical obstacles to a deep dissection in the anterior triangle are considerable. On the other hand, through an incision along the posterior border of the sterno mastoid, any region between the spine behind and the sagittal axis of the larynx and trachea in front, may be expeditiously exposed. If the cutaneous branches of the cervical plexus have to be cut, their sacrifice involves no great disability. The only important structure to be considered is the spinal accessory nerve. This nerve passes into the sterno-mastoid muscle on its deep surface at the junction of the upper and middle thirds of its anterior margin, and emerges opposite a point just above the middle of its posterior margin. The nerve is gently held upwards and backwards by a wide retractor. Another retractor pulls forward the sterno-mastoid muscle and with it bodily the contents of the carotid sheath and the prevertebral layer of fascia. Blunt dissection through this fascia exposes without further ado the pharynx, the posterior half of the larynx and the cervical œsophagus. During the operation 
the anæsthetist is well out of the way of the operator, and the anæsthetic may generally be given on an ordinary mask. Sometimes the operation entails pressure on, and displacement of the larynx or trachea; in such cases intra-tracheal ether may be given through an ordinary No. I2 gum-elastic urethral catheter. In any case, preliminary tracheotomy or laryngotomy is not often necessary. Lastly, the remaining scar in the neck is almost, if not entirely, hidden by ordinary clothing. These points of advantage, which are well exemplified in Case 37, should lead us, everything else being equal, to operate in the posterior triangle.

Wounds of the Throat in General.-In time of peace, wounds of the throat are comparatively frequent, whether suicidal, homicidal or accidental, and they quite often result in injuries to the larynx, trachea, and great vessels. In war, they form, on the whole, a very small proportion of the wounds of the various parts of the body. Delorme puts it at about $I \cdot 2$ per cent., but unfortunately with a mortality of I2 per cent., and this not including those who in all probability die on the spot from injuries to the great blood-vessels before they come under the notice of the surgeon. It is remarkable, however, that the great vessels and nerves often escape injury in perforating or penetrating gunshot wounds of the neck (see Case 30).

This last fact is explained by the mobility and elasticity or plasticity of the constituent structures. The larynx or trachea, for instance, seems to slip 
out of the way in a most wonderful manner (as in Case 38). Koerner narrates a case in which a ball passed behind the larynx, producing merely a temporary immobility of one vocal cord from contusion of the posterior crico-arytenoid articulation, in its transit. In a case, on the other hand, in which the anterior part of the larynx was shot away, the thyroid cartilage had already, owing to age, undergone ossification, and had, therefore, lost its normal flexibility and become brittle. Canuyt points out that the larynx is protected by the chin, especially when the soldier is lying on his face, and that in many cases it only escapes at the sacrifice of the inferior maxillary bone, which is often damaged in war (see Cases 24, 25, 27, 28, 29, and 30).

The fact of the larynx being injured may be obvious from the direction of the wound, from the appearance of the parts lying exposed, and from the expectoration of blood with disturbance of voice, but mainly from the passage of air during respiration.

Canuyt gives the following description of various disturbances by which wounds about the larynx may manifest themselves :

(a) Respiratory Disturbances.-These may vary in intensity from the slightest difficulty in breathing to dyspnœa or even asphyxia such as to call for immediate tracheotomy.

(b) Phonatory Disturbances.-These may vary from partial aphonia or hoarseness up to absolute mutism. The causes of these are many, being sometimes R.G.W. 
endolaryngeal, as, for instance, involvement of the thyro-arytenoid muscle, or extra-laryngeal, as an injury of the recurrent nerve. It is well to keep in view the share taken by the various muscles in the production of voice. This is dependent not alone on the intrinsic muscles, but also on the cricothyroids which lie close under the skin and, arising on the anterior surface of the cricoid cartilage, pass upwards and backwards in the form of a fan to the lower margin, and the external and internal surfaces, of the thyroid cartilage. Injury of these muscles is sufficient to cause such disturbance of voice as might lead one to suppose that the larynx had been penetrated even when this had not taken place. (One muscle was probably injured in Case 35.) Lastly, loss of voice and especially deaf-mutism are often attributable to emotional or neuropathic paralysis (traumatic hysteria).

(c) Emphysema.-This ought to be a frequent occurrence at the front, as a fracture or fissure of the larynx must from time to time give rise to a generalized emphysema, terminating rapidly in death. It is, however, extremely rare at stationary hospitals, and, indeed, one of us during three months' continuous service, which included the heavy influx of casualties resulting from the Ypres fighting, did not encounter a single case of this condition.

Non-penetrating Wounds.-Occasionally a bullet may strike the exterior of the larynx without penetrating, and damage mainly the external surface of the box and the more exposed muscles. 
Koerner describes a case in which a shot entering on the right side of the larynx through the lower jaw, injured the posterior part of the right half of the thyroid cartilage, producing a lasting fixation of the right crico-arytenoid joint.

In all cases of any severity there is evidence of perichondritis, as indicated by thickening of the wings of the thyroid cartilage, with tenderness on pressure (Case 35).

Penetrating Wounds.-For penetrating wounds which do not perforate right through the larynx, we have to go back to comparatively ancient history. Canuyt quotes a case of Traube's in which a projectile in the cervical region remained quiescent for twelve years, at the end of which time it was extracted by the endolaryngeal route. On laryngoscopic examination the writer found the left pyriform sinus completely hidden by a foreign body which was very hard to the probe. He was able to remove it with laryngeal forceps and it turned out to be a bullet measuring a quarter of an inch in diameter and half an inch in length.

Another case is the classical one of Fauvel's, in which a bullet had lain for ten years and was found in the larynx. The projectile entered below the left eye in the war of 1870 , and though the patient lost a good deal of blood and experienced considerable pain for several days in the region of the left eye, no further symptoms presented themselves until I880, and in January I88I he consulted Dr. Fauvel on account of pain in the throat of the duration of seven months. At first the pain was experienced in 
both tonsils, but later it was localized in the lower jaw at the level of the temporo-maxillary articulation and the left ear; he then commenced to cough and felt pricking pains in the larynx, especially on the left side. He even expectorated a little blood and the cough became frequent, especially at night. The patient thought that a fishbone must have stuck in his throat, and he kept trying all the time to swallow it or to pull it out with his finger, with which he said he could touch some hard and resistent body. Some days later the voice became weak and hoarseness was rapidly replaced by aphonia; this was later accompanied by difficulty in swallowing. On laryngoscopic examination the epiglottis and the right half of the larynx appeared perfectly normal. In the left half, however, there was seen a 'blackish tumour', irregular in shape and filling the whole of the left half of the infundibulum of the larynx, hiding the aryepiglottic fold, the ventricular band and the posterior three-quarters of the left vocal cord, and filling up a part of the hyoid fossa. The appearance suggested at first a melanotic cancer or tuberculous disease of the larynx, but no improvement took place under treatment, while the tumour became larger and in particular the aryepiglottic cold became the seat of a considerable degree of œdema. The condition seemed to be an osseous tumour. Dr. Fauvel tried several times to remove it but without success, and proposed operating again on the following day. However, the patient, after a sleepless night spent in coughing and spitting, 
was attacked with nausea and vomiting and during a violent effort ejected into his hand a hard heavy body. On examination he was astonished to find that it was the bullet, which he had certainly never expected to find in this place.

These cases show that the migration of projectiles is not a mere fancy, and above all that their presence may only be revealed a long time after the wound, when they cause accidents of considerable seriousness, and all the more because the practitioner may be consulted without being informed as to the occurrence of the wound and may find himself greatly at a loss. They would suggest the advisability of removing the projectile whenever possible and, if extraction is impossible, of warning the patient as to the possibility of it causing disturbances even at a very distant time; so that he may not fail to inform his medical adviser of the circumstance and enable him to attribute them to their true cause.

Perforating Wounds.- These may pass through the larynx, causing comparatively little damage, but this is very exceptional. Such a case is reported by Sir Anthony Bowlby, that of an officer who was wounded by a Mauser bullet which passed transversely through the thyroid cartilage just below the vocal cords. This resulted in the immediate coughing up of some blood and a feeling of suffocation which, however, did not last long. Afterwards he had loss of voice for some days and then a period in which the voice was hoarse and phonation painful. Eventually complete recovery took place. 
Moure and Canuyt narrate one in which the bullet entered the left side of the neck to escape on the right. The larynx was felt to be the seat of a slight perichondritis, and on laryngoscopic examination the left ventricular band was red, swollen and ulcerated, and covering the vocal cord. It was probable that the thyroid cartilage had been perforated, but the condition settled down and eventually the patient recovered completely.

More often these wounds cause such interference with respiration as to necessitate tracheotomy in order to save life, as in Case 26. Occasionally recovery takes place and it is possible to remove the tracheotomy tube, but experience has shown that this must not be done until it has been made certain that the improvement is not merely a temporary subsidence of the obstruction, but a lasting one. At this stage intubation may be considered as a possibility.

In other cases a stenosing cicatrization takes place and the tube may have to be retained for life unless laryngostomy or tracheo-laryngostomy is successfully carried out. In some of these cases the larynx has been damaged and broken up so that its lumen has nearly or entirely disappeared and been replaced by cicatricial tissue.

In one of Moure's cases the bullet had passed through the larynx. Edematous infiltration of the left ventricular band produced such a degree of asphyxia that the patient very nearly died owing to his having refused to submit to tracheotomy. Eventually the operation was carried out, and though 
certain complications, including emphysema, took place, the patient was in good condition ten days later, and a fistula, which was present, had dried up so that there was every prospect of removing the cannula. On the other hand, in another case a perforating wound of the larynx resulted in acute œdema requiring tracheotomy. The tube was removed a week later, but difficulty in respiration gradually reappeared and on laryngoscopic examination, although the upper part of the larynx appeared absolutely normal, there was below at the entrance of the trachea a fibrous diaphragm which closed up the laryngo-tracheal tube to a great extent. Indeed, it produced a narrowing such as would scarcely admit a probe of the diameter of a pen-holder. The patient was only in comfort when at rest and he was obviously a subject for tracheo-laryngostomy.

In another case complete laryngeal stenosis followed a perforating wound; intra-circo-thyroid laryngotomy was hurriedly performed but a genuine tracheotomy was called for, and subsequently Professor Moure carried out the operation of laryngostomy.

Injuries to Nerves.-The development of the signs indicative of paralysis of vocal cords leads to the inference that the vagus or recurrent nerve has been damaged by the projectile, though whether the nerve has been cut through, severely bruised, or simply considerably concussed may only be decided by the subsequent course of events. At first the voice and the power to cough may be almost normal, but afterwards-sometimes with a waste of air every 


\section{I04 INJURIES OF NOSE AND THROAT}

time it is used-the voice may become somewhat weak and husky. The true indication is, of course, the appearance on laryngoscopic examination, when the cord is found at first to be in the middle line, but later in the cadaveric position-about halfway between the position of phonation and that of quiet inspiration. Dyspnœa may be present to a slight extent at the commencement of the paralysis, but if it is very considerable there is great probability that the damage to the nerve is accompanied by injury to the structures of the larynx itself.

A slight irritation of the recurrent nerve may show itself by a partial aphonia with a spasmodic cough.

If the nerve is simply bruised, the symptoms are transitory and pass away gradually. If, on the other hand, it has been cut completely through, the conditions persist and no improvement shows itself.

In a case of paralysis of a vocal cord, the question arises as to whether the damage is to the vagus (or spinal accessory fibres) or to the recurrent nerve, and we may be guided to some extent by the direction of the projectile. The probability of the pneumogastric being struck without damage to the carotid or jugular vessels which protect it, is comparatively unlikely; whereas the recurrent laryngeal lies in a slightly more anterior plane and sufficiently far from these vessels to be accessible to the projectile without their being damaged.

Cases are, however, seen in which with paralysis of a vocal cord the projectile has taken a course quite away from this nerve, in which case we have to think 
of a lesion of the pneumogastric, or it may be of the spinal accessory, as, for instance, if the route of the projectile has been obviously above the level of the thyroid cartilage.

The following case is one of the rare instances in which the bullet has apparently injured the vagus nerve ; for it we are indebted to Sir Anthony Bowlby.

'W. S., private in the Coldstream Guards, was hit while in the prone position at Magersfontein. The bullet (Mauser) entered the right orbit and destroyed the eye. It then traversed the superior maxilla and entered the mouth through the soft palate, and afterwards passing out of the pharynx below the tonsil, emerging from the neck just behind the posterior border of the sterno-mastoid muscle, to re-enter the neck above the clavicle, which it smashed, and then passed away. In its passage it must have divided or seriously injured the vagus nerve inside the carotid sheath, for the vocal cord on that side was completely paralysed and did not recover. The great vessels of the neck never showed the slightest evidence of injury.'

Canuyt describes a case of Dr. Moure's in which there was paralysis of the left vocal cord almost in the median position without swelling or redness of the neighbouring parts, and in which the projectile penetrating at the anterior border of the sternomastoid had apparently passed between this muscle and the thyroid cartilage to disappear somewhere or other. By radioscopy it was found that a triangular portion of shell lay at the level of the third dorsal vertebra close to the œesophagus. In view of the 
depth of its situation and the improvement which gradually took place, it was left in position. At the end of two months the patient seemed to have recovered, and the paralysis of the vocal cord had completely disappeared. In this case it was probable that the left recurrent was not cut through, but only severely contused.

The impact of a bullet which traverses the laryngeal box from side to side may rotate it bodily on an anteroposterior axis. The laryngoscopic image will then convey on the side tilted higher a false impression of impaired adduction of the true cord, because the false cord obscures it by being tilted inwards; and on the side of the larynx which is tilted lower, the position of the cord below that of the opposing cord will suggest an erroneous idea that the crico-thyroid muscle in that side had been damaged. In Case 34 the appearances suggested these two lesions, neither of which were really present. Contrast Case 35, where a careful study of the evidence showed the simultaneous existence of both these injuries; that is to say, there were impaired adduction of one cord and paralysis of a crico-thyroid muscle on the same side.

The above is an instance of the sort of diagnostic difficulty which is encountered in dealing with intralaryngeal wounds. But a problem which occurs much more frequently is presented by any case which shows only defective mobility, or slightly abnormal position, of one cord or one arytenoid, as in Case 35. In this and similar cases we have to decide whether the disability is due to gross injury to the cricoid or 
arytenoid cartilages ; or to one or more muscles ; or to a nerve. We have already alluded to the anatomical improbability of the vagus or recurrent laryngeal nerves being injured without gross injury to neighbouring important structures. Here it needs only to be added that a vagus injury will manifest itself, concomitantly with the laryngeal signs, by an acceleration of the heart-beat and some irregularity of respiration. Whereas, if only the superior laryngeal branch of the vagus is damaged, there will be hemianæsthesia of the larynx, which, with the aid of a laryngeal mirror and probe, and a good head-light, is not difficult to demonstrate.

Diagnosis.-The existence of a wound of the larynx or trachea may be established by the position of the wound, the occurrence of loss of voice, stridor, the escape of air either outside or into the tissues, or recognition of some laryngeal structure in the wound; and in some cases by the results of X-ray examination.

We have above alluded to the anatomical position of the larynx and trachea, but we have also shown that these structures may glide out of the way of a projectile.

The escape of air through the wound on expiration, or its entrance on inspiration, are obviously decisive.

Loss of voice, along with the above-mentioned signs, would add to the probability. As has been seen above, it may result from an injury which does not necessarily penetrate the larynx. Moreover, loss of voice may result from disturbance of the central nervous mechanism for speech, as we have seen, and 
in the absence of signs of a wound of the larynx, may be readily diagnosed, more especially if the loss of voice and speech is so complete that not even a whisper is uttered (Apsithyria). See Case 36 .

The case is somewhat more obscure if with a slight wound in the neighbourhood of the larynx there is associated complete loss of speech and voice, of central origin. Here, of course, the evidence of the laryngoscope, when its use is possible, would decide as to the local or central origin of the loss of voice. We have described conditions in the larynx produced by penetrating as well as by glancing wounds, and also paralysis of a vocal cord from implication of the motor nerves to the larynx.

Dyspnœa of laryngeal character, i. e. more marked on inspiration, would suggest narrowing of the lumen of the larynx, if there exists an injury in its neighbourhood.

Exposure of the wounded structures would appear to be the most obvious sign; cuts through the thyroid cartilage, for instance, would be unmistakable. At the same time, in the midst of the hæmorrhage and the general condition of pulpification of parts, the state of the structures may not be obvious to the eye. In Case 26, for some time no landmarks could be identified.

An X-ray photograph will, in good hands, display the outlines of the larynx sufficiently for recognition, the main localizing landmarks in the profile picture being the body of the hyoid bone and the posterior part of the cricoid cartilage. Under normal circum- 
stances the anterior outline of the thyroid and of the cricoid should be unmistakable, and obliteration or haziness would indicate that the cartilage had been damaged. A case of Denker's illustrates the use of the X-rays, though their value in most instances is overshadowed by other more obvious evidence of laryngeal obstruction, and by the condition of the parts revealed at operation.

The expectoration of blood on coughing is almost invariably present in wounds damaging the interior of these air channels.

Injury to the pharynx or cesophagus may be assumed when there is an escape of food or liquid during attempts at swallowing or drinking (Case 34). The same may be said of the escape of salivary secretions.

Injury to the laryngeal nerves is indicated by an irritable cough or by loss of voice reduced to a whisper (aphonia as distinguished from apsithyria), and by the appearance of paralysis of a vocal cord on laryngoscopic examination.

Injury to the neighbouring vessels would be indicated by the signs applicable to wounds of such structures in other parts of the body. Naturally damage to the main vessels usually leads to death before aid can be afforded, but a very considerable amount of hæmorrhage may take place when only the branches or tributaries are involved. Injuries of the external jugular veins are probably those which come most frequently under the notice of the surgeon in civil life. In warfare, wounds of the great vessels 
may show themselves by the formation of traumatic aneurysm with rapid swelling. Occasionally also aneurysmal varix or varicose aneurysm may occur, the former being indicated by the purr and thrill.

The presence of a projectile or other foreign body was formerly sought for as a matter of routine by means of probes of various kinds, one of the most ingenious being Nilaton's porcelain-tipped one, which became blackened when in contact with the uncoated leaden bullet then in vogue. At the present time probing is avoided as much as possible, unless when, at a later stage, the obstinacy of the symptoms or the absence of definite X-ray information makes its use imperative. At the present day it is a rule to have an X-ray examination made. At the same time external palpation and examination of the mouth and throat by means of reflected light and the laryngoscopic mirror, or the bronchoscope, afford us information which in some cases will prove of the utmost value. The presence of a projectile may be forced on our attention by the persistence of suppuration or the obvious involvement of a nerve in producing sensory or paralytic signs. The usefulness of endoscopic methods is illustrated by Case 38 .

Treatment.-Wounds of the throat, when seen on the field of battle soon after their infliction, call for first aid, carried out on general principles, and in the absence of unmistakable evidence of injury of the pharynx, œsophagus, larynx, or trachea, should be treated like those of other parts of the body, by such cleansing and stoppage of bleeding as is practicable. 
In most instances it is confined to the application of a 2 per cent. solution of iodine in alcohol to the skin round the wound, even allowing some of the liquid to pass into the wound itself; the soldier's first fielddressing is applied with a bandage pinned tightly round it. If there is a wound of the larynx or trachea it is important that the head should be bent well forward so as to bring the chin as nearly as possible to the sternum, as was done in Case 26. In the first instance this may have to be carried out simply by means of pillows and props, the appropriate bandages being applied at the first available opportunity.

Bleeding from the great vessels or from their branches can for the time being only be influenced by digital compression. This may have to be carried out by relays of bearers, though much may often be done by means of a pad kept tightly in position by a bandage carried round the head and the axilla. This may have to suffice until it is possible to explore the wound sufficiently for ligaturing the bleeding vessels above and below, or, as a last resort, ligaturing the main trunk.

If the wound involving the larynx gives rise to severe dyspnœa, it may be necessary to open it up and insert a tube. Under favourable circumstances a planned tracheotomy is desirable, but in the first instance a tube may have to be introduced wherever practicable, and inter-crico-thyroid laryngotomy may often be carried out when circumstances render it impossible for tracheotomy to be performed. It is to be understood, however, that the cannula should be 
removed as soon as it is possible for a genuine tracheotomy to be carried out.

Denker has devised a triangular-bladed lancet guarded by shoulders and mounted on a firm handle, with which the skin and soft tissues over the cricothyroid space can be instantly punctured with the minimum risk of damage to the crico-thyroid muscles ; into the puncture thus made a tube on a pilot can be rapidly introduced. An instrument of this description would facilitate the carrying out of the operation on the part of the regimental medical officer, though the methodical as well as the improvised methods are quite familiar to him.

At the dressing-station a great deal more can and ought to be done.

As an obvious general surgical rule, it will be admitted that the cleaner a wound is the less often should it be dressed or plugged. And such bullet wounds as are obviously clean may be interfered with as little as possible between the dressing-station and base hospital in France, when the patient's transit is to be immediate. But few rifle wounds are clean; a shrapnel bullet usually produces a ragged septic wound; while jagged pieces of shrapnel or bombcasing, time-fuses, and similar pieces of metal have a lower velocity than a bullet, and also penetrate by a flat surface rather than by a point. These frequently carry before them into the patient's body mud, shreds of khaki, or anything else which happens to be covering the part hit. Wounds of this sort about the throat should, therefore, be cleansed, redressed, and replugged 
at the field-ambulance and the clearing-station, even though they may be going on to the base hospital in a few hours. If at the dressing-station there be found any definite reason for leaving the wound alone until the patient reaches the base in France, such as, for instance, a large wound into the larynx or trachea, or hæmorrhage which recurs violently every time a plug is removed, a label should be pinned on the bandages, bearing a brief statement of the facts. It will then be expected that he will be sent through as soon as possible without redressing. The head must be fixed in a flexed position.

If the pharynx or œsophagus be opened, an œsophageal tube should be introduced, and the most convenient method is through the nostril (Case 30) ; for this it is desirable to find out which is the wider of the two nostrils. In some cases the wound is so large that a stomach-tube may be introduced through it and retained. Until this is done no food should be given except in the form of nutrient enemata. If a larger tube be used, and passed through the mouth, it is difficult, either by strapping, pinning to bandage, or any other method, to keep it in place. It will be either coughed up, vomited up, or deliberately pulled out by the patient (Case 37). It is, however, usually possible to teach him to pass it on himself (Case 24).

In the later stages of the history of wounds of the throat, when sepsis has been checked or greatly reduced by means of cleansing and non-irritating antiseptics, healing is apt to be complicated by the formation of unfavourable cicatricial contraction.

R.G.W. 


\section{II4 INJURIES OF NOSE AND THROAT}

Projectiles and other foreign bodies, if they are easily accessible, should be removed, more especially if their presence is causing serious disturbance; and here, as in other parts of the body, it is necessary to consider whether the interference for the purpose of removal may not be dangerous and more damaging than the mere presence of the projectile itself. If, as we have said before, its presence leads to the continuance of suppuration or to pain or loss of function in nerves, and if, at the same time, it can be absolutely located and reached by the surgeon, its removal is indicated. If, however, the only evidence of its presence is the fact of its being revealed by X-ray examination, then, if it is not easily accessible, it ought to be left in situ, unless likely to ulcerate through into important adjacent structures. There should, however, be the proviso, to which we have before referred, that in the case of the subsequent occurrence of symptoms which are not obviously and easily explained, the possibility of the foreign body having shifted its quarters and aroused inflammatory reaction in its new situation should be kept in mind by the patient, and the history of the wound should be communicated to whoever is in medical charge.

Again, we have to keep in mind that the data afforded by the X-rays must be judiciously interpreted and that the possibility of being misled by them owing to a slight error in the position of the plate or the shifting of the foreign body in the interval between the taking of the photograph and the carrying out of the operation, must be kept in mind. 
In Case 36, the foreign body had in this interval slipped down one inch, and in one of Denker's cases, owing to the obliquity in the application of the plate, the projectile appeared to be close to the mastoid process, whereas in reality it was situated deeply in the sub-maxillary region. In several cases a distinguished surgeon sought, under the guidance of the X-rays, for the projectile in the side of the neck, and failing to find it he made a further external examination and felt it under the skin considerably further back, in a position from which he was able to extract it without any difficulty.

Mackenzie Davidson's original method of localization has been modified by others but has scarcely been improved upon.

The carrying out of operations on the X-ray table is a process which affords great promise, and the recently published method of noting the position in which prodding with the finger or probe produces the maximum movement of the foreign body when observed through the X-ray screen, seems very convincing. Although its deviser considers that its use is chiefly applicable in the thigh, it seems as if it ought ${ }^{\circ}$ to be of value in the throat and neck as well. 


\section{ILLUSTRATIVE CASES}

Nose.

No. I. PTE. B.

Bullet wound. Entrance: tip of nose, tearing off right lateral cartilage of aperture. Then through right maxillary antrum. Exit: just above apex of right mastoid process.

The bullet, in passing through right nasal orifice, bent the quadrilateral septal cartilage to the left, and crushed the anterior half of the right inferior turbinate bone to pulp.

\section{No. 2. Sergt. O.}

Bullet. Entrance : left side of nose, at a point midway between columella and inner canthus, half inch from bridge of nose. Exit: $I_{\frac{1}{4}}$ inches vertically below right mastoid apex.

In its course the bullet traversed the right antrum, and fractured the right lower jaw near the angle.

Nose. The septal cartilage had been perforated by the bullet; the lower horizontal margin of the perforation was adherent to the left inferior turbinate. There were no signs of damage to the right antrum.

Treatment. The anterior half of the left inferior turbinate was removed.

No. 3. Wounded German Prisoner, Pte. F.

Shrapnel. Entrance: left ala nasi, separating upper lateral cartilage from nasal process of superior maxilla. The bullet then splintered the outer wall of 
left antrum and emerged just above angle of left lower jaw.

In hospital the nose was kept in good position, and healed without any deformity or nasal obstruction.

\section{No. 4. Pte. B.}

Shrapnel. Two wounds (I) above right eyebrow, and (2) over right nasal bone.

Globe of right eye ruptured. The oculist (Mr. W. B. Oliver) advised against its removal on the ground that the collapsed globe would make the best stump for a glass eye.

Skiagram. Scattered shrapnel seen (I) one inch above right frontal eminence and (2) over right nasal bone. Frontal sinus apparently normal.

Treatment. No operation indicated. Sent home well. The small fragments of shrapnel, probably spent, had neither fractured the bone in any situation, nor set up any material degree of suppuration.

\section{No. 5. Pte. A.}

Shrapnel. Right temple two inches behind outer canthus, and over right nasal bone.

Globe of right eye partly collapsed.

Skiagram. Scattered shrapnel right side of face, mostly over nasal bone and nasal process of superior maxilla. No fracture.

\section{No. 6. PTE. H.}

Shrapnel. Shallow ragged wounds of left side of nose, left cheek, and left temple.

Skiagram. Scattered small pieces of shrapnel seen over left nasal bone, left superior maxilla opposite the last molar tooth, and just behind external angular process of left frontal bone. No fracture existed. 


\section{Antrum.}

\section{No. 7. Sergt. L.}

Shrapnel. Entrance: immediately below left zygoma just in front of coronoid process- of left lower jaw. Exit: through the right outer canthus.

Eyes. Right lobe collapsed; left can count fingers only.

Skiagram not waited for.

Left antrum explored by both Caldwell-Luc and nasal routes. It contained muco-pus. The floor of the left orbit was missing, and found in fragments in the left antral cavity. A drainage-tube with lateral holes was passed below the cheek, through the antrum, into the nose, and out at the nostril. Comminuted ethmoid galleries were removed intra-nasally on both sides with a curette. The posterior part of the mesial wall of the right antrum was found fractured and purulent ; so drainage to the antrum on this side also was obtained by removing a small piece of the anterior end of the inferior turbinate, and making a hole the size of a threepenny-piece in the antral wall at this place.

The patient was sent home convalescent.

NotE. No shrapnel was found, and it was believed that the projectile, whether bullet or shrapnel, had passed right through. It was considered to be certainly shrapnel, partly from the patient's definite statement, and partly from the amount of shattering produced. The extensive comminution of the orbital plate is very often found in shrapnel wounds of the antra, especially on the side of entry. Compare No. 9, and contrast No. I2. From the course taken it seems hardly possible that the shrapnel could have passed behind the posterior edge of the vomer; but no lesion of the septum could be discovered. 
No. 8. Sergt. S.

Bullet. Transversely from left to right through both antra, opposite the second molar tooth.

Nose. Normal. Post-nasal space. Normal. Sent home well.

NOTE. For a bullet thus to pass transversely through both antra, even though it be through the lowest and most posterior parts of these cavities, without touching the septum, must be very rare. But an examination of a series of skulls will show that it is just possible in a very few, in which the angle to the horizontal made by the posterior edge of the vomer is very acute.

\section{No. 9. Pte. B.}

Shrapnel. Entrance: two inches horizontally in front of left tragus. No exit wound. A probe could be passed transversely through left antrum, both ethmoids and into right antrum, finally stopping at right outer-antral wall. Below the level of the middle turbinate the septum nasi was median and normal.

On admission both eyes were entirely closed by œdema, and there was extensive hæmorrhage from nose and mouth.

Skiagram not waited for.

Operation. External excision horizontally through entrance wound. Bleeding vessels tied. Comminuted left outer antral wall removed. A little pus was found. Orbital floor found comminuted ; from it pieces of bone were removed, the largest being approximately a circle of three-quarters of an inch diameter. Drainage through external wound, through antrum, and out at nose, by a tube with lateral slits.

Right side. Antrum explored by Caldwell-Luc route. Shrapnel and débris of bone removed. Eyelids fomented. Sent home well. 
Note. The operative treatment of this case exemplifies three different routes of access to the antrum. The left antrum was opened both externally from the wound in cheek and also internally through the nose; the right antrum through the mouth by everting the cheek from the maxilla (Caldwell-Luc).

\section{No. IO. GunNer W.}

Shrapnel. Two ragged wounds below left eye. Dead bone and small fragment of shrapnel removed through wound, from zygoma and outer antral wall. Purulent cavity irritated. Uneventful cure.

NoTE. In this case the zygoma received part of the charge, and thus no doubt diminished the damage to the antrum, so that no shrapnel actually perforated its walls.

\section{Hard Palate. \\ No. II. Pte. B.}

Patient did not know what the projectile was which hit him. Entrance: right cheek, $I \frac{1}{2}$ inches below centre of lower eyelid. No exit wound. Mouth : a smooth oval swelling, I inch by $\frac{3}{4}$ inch, and raised from the surrounding surface by $\frac{1}{8}$ inch, was found at the junction of anterior and middle thirds of the hard palate, a fraction of an inch to the right of the middle line; this swelling was firm but not hard, and except that it was very tender, exactly resembled a submucous gumma which had not yet ulcerated.

Skiagrams showed what appeared to be a bullet impacted in the upper, nasal aspect of hard palate.

Operation. The 'bullet' could be felt firmly impacted in the floor of the right nasal fossa, but by no means could it be removed therefrom.

An antero-posterior incision was now made through the muco-periosteum of the hard palate from a line 
joining the first upper molars to within $\frac{1}{2}$ inch of the central incisors. The 'bullet' proved to be the screw-top of a time-fuse, as large as a sixpennypiece, and $\frac{1}{6}$ inch thick. The muco-periosteum was replaced and stitched with horsehair. An alkaline nasal douche was used three times daily. In twelve days the douching fluid ceased to come through the floor of the nose into the mouth ; the hole had healed over.

Transillumination showed no abnormality of the right antrum, which was, therefore, not interfered with. Sent home well.

Note. This case showed that a projectile may traverse an antrum without inducing suppuration, and that the best route for removal is not always the one suggested by the skiagram.

\section{Antrum. Rotation of Bullet.}

\section{No. I2. Pte. G. (Fig. 8.)}

Bullet. Entrance: just below right inner canthus. No exit. Left cheek swollen, tense, and shiny. Both eyelids of both eyes œdematous. Right eye cannot be opened at all. Left, vision good. Constant slight epistaxis.

Transillumination. Both antra opaque. No infraorbital crescent of light, illumination of left fundus (the only one available), or subjective sensation of light.

Skiagram. Bullet seen impacted in postero-external corner of right maxilla, just behind and above second molar tooth. The bullet was pointing the wrong way, i.e. point inwards, base outwards. The third molar tooth was missing.

Operation. The crushed anterior ends of both inferior turbinates were removed. The septum was 


\section{I22 INJURIES OF NOSE AND THROAT}

too badly damaged for immediate correction, and was left alone. Nose irrigated with alkaline douche and lightly plugged with ribbon gauze, which was removed twelve hours later.

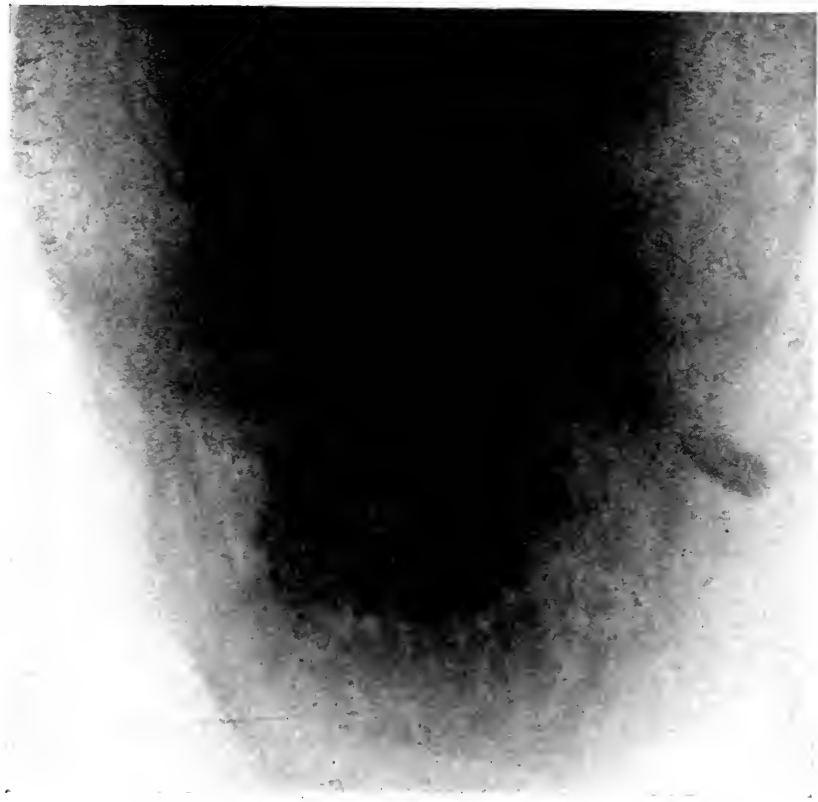

Fig. 8. Case No. iz.

The right orbit was full of blood-clot; there were no remains of the globe ; this cavity was septic, and dressed by a wick of ribbon gauze, soaked in hypertonic saline, frequently renewed. The right inferior tarsal plate was loose, and together with bone débris just below the infra-orbital ridge, was removed. The 
right upper eyelid was found to be lacerated, and was repaired. The left antrum was approached by the Caldwell-Luc route, and from the pus in its interior pieces of bone were removed, together with the third molar tooth, which was lying loose in the cavity. The left orbital floor was intact. The accidental discovery of crepitus showed that the left jaw was fractured (this was not evident in the skiagram). The bullet was too far back to be accessible under the cheek, and was removed by a small external incision. A tube with lateral openings was passed through left nostril into left antrum, under the cheek and out of the left angle of mouth. Sent home well, but badly scarred.

Note. In this case the projectile traversed the antrum obliquely, not far below the orbital floor, which was, however, not damaged. But it should be noted that the projectile was a bullet, not shrapnel, and the antrum which it traversed was on the exit, not the entrance, side. Contrast Case 9.

\section{Escape of Septum Nasi.}

$$
\text { No. I3. PTE. P. }
$$

Bullet. 'On admission both cheeks swollen. Epistaxis from right side. There was a black eye on the left, and a triangular subconjunctival hæmorrhage over the outer part of the sclerotic. Entrance: transversely through left cheek immediately below the zygoma and just escaping the anterior border of upper jaw. Exit: at a corresponding point on the right side. Nose: posterior part of the septum bruised, covered with pus on both sides. Post-nasal space: muco-pus in the left fossa of Rosenmüller and blocking the left Eustachian ostium. The septum on its posterior edge, about half-way up, had a small piece chipped out of it. 
Treatment. Alkaline nasal douche, and, in view of the subconjunctival hæmorrhage, we awaited the possible onset of signs indicating that a fissured fracture has extended to the anterior cranial fossa.

Subsequent history : nothing alarming supervened, and recovery was uneventful.

\section{Antrum. Tetanus.}

No. I4. PTE. R.

Shrapnel. Entrance: by a vertical gutter-wound one inch long, extending downwards from the middle of left lower eyelid. Obvious crepitus between here and left outer canthus.

Left eye sees light; cannot read or count fingers.

Skiagram (lateral view only). Shrapnel seen (I) in left antrum, (2) opposite upper second and third molars.

Operation. External incision from the upper end of gutter-wound, along outer half of infra-orbital margin. No shrapnel found. Dead bone, partly from outer antral wall and partly from orbital floor, removed. The antral cavity was purulent. A hole of $\frac{1}{4}$ inch diameter was made with a burr below anterior end of left middle turbinate. A tube with lateral holes was passed via the nostril through this hole, through the antrum and out just beyond the outer canthus. The shrapnel was now discovered in two pieces lying free under the mucous membrane of the left cheek, and was removed.

Two small stitches united the tear in the lower lid, on its conjunctival aspect, with the object of avoiding subsequent ectropion.

Eight days after the operation, and fourteen days after he received his wound, he complained of anorexia. 
All the neck muscles and the masseters became increasingly stiff.

Treatment. Anti-tetanic serum, I,500 units, subcutaneously; 20 c.c. of 2 per cent. carbolic acid subcutaneously. The wound to be frequently irrigated with sea-water. From this day the serum was repeated twice on the third and fifth day. The saline was continued, and the carbolic injections repeated, every day. Three days after its development, the muscular stiffness began to diminish. He was sent home well.

Note. As regards the tetanus, it will be noted that the latent period was fourteen days. Had the lateral skiagram been supplemented by others in a frontal or oblique plane, the shrapnel would probably have been found more quickly.

\section{Antrum. Septum Nasi.}

$$
\text { No. I5. PtE. D. }
$$

Bullet. Entrance: one inch to the outer side of middle of right nasal bone. Exit: just above angle of left jaw. The bullet traversed both antra and the bony septum nasi. It narrowly missed the left upper alveolus and teeth, and emerged just in front of the angle of the left lower jaw, between the anterior margin of the ascending ramus and the last lower molar tooth.

Operation. Caldwell-Luc approach to clear left antrum of dead bone. The fissured septum nasi was forcibly straightened and splinted each side with a short tube.

Subsequent history. Edema of the cheek, which yielded to fomentations, and a very acute rhinitis with a temperature of nearly $102^{\circ}$, treated by alkaline douches. Sent home well. 


\title{
Perforating Wound of Antrum. Spontaneous Recovery.
}

\author{
No. I6. PTE. F.
}

Shrapnel. Entrance: $\frac{1}{2}$ inch horizontally in front of left tragus. Exit: just to nasal side of right inner canthus. The left eyeball had been removed at the field hospital. There was chemosis of both eyelids.

Treatment. Beyond irrigating the shrapnel-track and the left orbit with hydrogen peroxide (5 volume) warm, and then with carbolic acid ( $I$ in IOo), and also using an alkaline nasal douche, nothing was done. He went home, convalescent, nine days after being wounded. The fact that during his stay at Boulogne his respiration-rate never rose above I2, and was usually ro, remained unexplained; there was no other evidence of raised intracranial pressure.

NoTE. Having regard to the course taken by the projectile, it is remarkable that we could not find any evidence of damage to the left lower jaw, or to the 2 nd or 3 rd divisions of the Vth nerve on that side.

\section{Ethmoid.}

\section{No. I7. PTE. D.}

Shrapnel. Entrance: two inches behind left external canthus. Exit: at right internal canthus. Nasal bridge œdematous. Both eyeballs collapsed; stone blind. Nose : the ethmoidal cell-galleries of both sides were pulped.

Operation. An external incision was made, having a double curve, following the line of the eyebrows and meeting at the nasal bridge. From here the soft tissues were turned up over the forehead, and down over the nose. The septum nasi had been entirely destroyed, except for a horizontal strip 
$\frac{1}{2}$ inch wide above the nasal floor. The entire lateral masses of the ethmoid on the two sides were cleared right up to the cribriform plate by the combined intra-nasal and extra-nasal routes. The frontal sinuses were found intact. The oculist (Mr. W. B. Oliver) advised leaving the globes for the better fixation of glass eyes on the collapsed globes as stumps. Sent home well, but blind.

NoTE. In cases where sufficient access to the upper ethmoidal regions cannot be obtained through the nose, or when it is necessary also to explore the frontal sinuses, an eyebrow incision, prolonged, if necessary, into the sulcus between nose and cheek. is most useful.

\section{Antrum. ? Tetanus.}

No. I8. PTE. H.

Patient uncertain whether shrapnel or bullet. Entrance : in centre of left orbital roof, $\frac{1}{4}$ inch behind eyebrow. No exit. Left eyeball unrecognizable.

Skiagram (lateral view only) showed two foreign bodies, one resembling a bullet, the other apparently an irregular piece of shrapnel casing, just beneath the orbital floor, abutting on the ethmoid. The surface of the body showed a uniform erythema, which was regarded as a tetanus antitoxin-rash, the serum having been given at a field hospital.

Operation. External incision below and parallel to the left infra-orbital ridge. No bullet or shrapnel found, but the nasal process of the left superior maxilla had been broken off and was lying loose in the upper and inner part of the left antrum. In shape and size it exactly corresponded to what, in the skiagram, had been interpreted as shrapnelcasing. Left orbital floor was completely gone. All dead bone was removed, and the wound drained 
by tubes with lateral holes, both externally and through the nose. On the fourth day after admission there was a sharp rise of temperature to $103^{\circ}$ without rigors. This was attributed to tetanus antitoxin disease. The erythema had disappeared.

Next day the temperature was I02. $2^{\circ}$, with dysphagia and a stiff neck. He was given I,500 units of anti-tetanic serum, which was repeated the following day, and the wound henceforth treated with hypertonic saline. The muscular stiffness abated three days after its onset.

But now the right eyelids and upper part of cheek became increasingly odematous. Another skiagram was taken, this time in a frontal plane, which showed a bullet deep to the bridge of the nose, just to the right of the mid-sagittal line.

Second Operation. A rifle-bullet was removed from below the right infra-orbital margin, by external incision.

Bacteriological culture (Sir A. Wright) taken from the left orbit, when first the neck stiffness appeared, although cultivated for 72 hours, was negative. Thirteen days after admission the patient was sent home convalescent.

NoTE. This case exemplifies, even more forcibly than No. I4, the fallacy of depending on one skiagram.

\section{Frontal Sinus.}

\section{No. I9. LCE.-CORP. J.}

Two shrapnel wounds. (I) Entrance: 2 inches vertically above right inner canthus ; (2) Entrance : over middle of nasal bridge, exposing the septum. No exit wound in either case. He was admitted with excruciating headache, necessitating morphia. He rapidly became comatose, with stertorous respiration. 
Before anything elaborate could be done for him, he died. It was found that the first of the two pieces of shrapnel, above mentioned, had passed through the right frontal sinus and lodged in the frontal lobe of the brain.

\section{Replacement of Fractured Maxilla.}

No. 20. PTE. N.

Bullet. Entrance: just above horizontal ramus of right jaw, close to angle. Exit: left cheek, $2 \frac{1}{2}$ inches horizontally behind ala nasi. There was on the right side a fracture of the hard palate, which was depressed into the mouth. Post-nasal space: the mucosa was tumid and inflamed, but showed no gross lesion. There were no other signs or symptoms whatever.

Treatment. An alkaline nasal douche was given, not because the nasal fossæ showed any lesion, but as routine. The only active treatment consisted in replacing the broken hard palate. Sent home well.

Note. It is difficult to understand why there was no evidence of injury of any of the important contents of the pterygoid fossa.

\section{Important Structures unhurt.}

NO. 2I. LCE.-CORP. S.

Bullet. Entrance : at the mid-point of a horizontal line, between the tip of the lobule of the right ear and the ligamentum nuchæ. Exit: through the left angle of the mouth; on its way the bullet perforated the soft palate and anterior faucial pillar, and knocked out the left lower first and second molar teeth.

Treatment. There were no symptoms. With R.G.w. 
ordinary surgical cleanliness, the wounded palate and fauces healed rapidly.

Note. It is unlikely that this bullet took a straight course from entrance to exit ; otherwise the important structures damaged would be too many to enumerate. We need only mention the internal carotid artery, the internal jugular vein, and the XIth and XIIth nerves.

\section{No. 22. PTE. J.}

Bullet. Entrance : 4 inches below the lobule of the left ear, along the posterior border of the sternomastoid muscle. Exit : just above the angle of the right jaw. Eyelids black; right cheek swollen; right eye ; a triangular sub-conjunctival ecchymosis on the outer side. No epistaxis. Mouth : the bullet had passed through the lower part of the left tonsillar fossa, and the tonsil was hanging by its upper pole. When the odema of the right cheek had subsided, there was a manifest right facial paralysis affecting the mouth only. Home convalescent.

Note. The left internal carotid was missed.

\section{Jaw.}

No. 23. PTE. A.

Bullet. Entrance : through anti-helix of left ear. Exit: through vertical ramus of right jaw, $\frac{1}{2}$ inch below coronoid notch. Exit wound extremely purulent. (Right otorrhœa and deafness ; hearing tests taken.) Post-nasal space: no sign of any gross lesion.

Skiagram. Comminuted transverse fracture of right lower jaw, below exit wound.

Operation. External horizontal incision. Dead pieces of bone were removed from the posterior border of the insertion of the internal pterygoid 
muscle, down to the lingula. Inferior dental nerve found intact. Drainage by tube. Jaw-bandage. Sent home with only slight limitation of power to move mouth.

\section{Mouth, Lower Jaw.}

\section{No. 24. (NAME, ETC. UNKNown.)}

Shrapnel. Large gaping wound lower part of face in middle line. The chasm extended from just above the upper lip to the upper border of the thyroid cartilage. The anterior half of the horizontal ramus of the jaw on both sides was pulped. The hyoid bone was comminuted, and the tongue immobile. The whole floor of the mouth was herniated. During a journey of 30 hours, in a hospital train, he had fed himself through a self-passed rubber-tube, ro inches by $\frac{5}{8}$ inch.

Treatment. After I4 days' incessant cleansing, the wound did not clean up sufficiently for a plastic operation or the insertion of a prothesis; so the patient was sent home.

\section{No. 25. PTE. G.}

Shrapnel. Gaping wound from the left lower lip down to the hyoid bone. There was not a great loss of tissue ; the condition rather suggested that a deep vertical incision through soft tissues and bone had been made. The left lower jaw, with the soft tissues covering it, gaped from its fellow like a half-open door; the wound was not at all septic; the floor of the mouth on this side faced straight forward instead of upward.

Skiagram. There was a simple fissured fracture through the horizontal ramus of the right lower jaw, just in front of the angle. The left jaw, from the symphosis backwards for $\frac{3}{4}$ inch, was comminuted. 
Operation. The fracture on the right was wired; the comminuted bone on the left, with the two incisor teeth, was removed; the ends of the broken jaw on this side were brought together and wired. Plastic operation on lip, cheek, and chin. One drainage tube was inserted, behind symphysis menti into mouth. Eleven days later the tongue was fairly movable, and speech was distinct. The jaw had undergone shortening only in an antero-posterior direction; so that while the upper incisors and canines lacked opponents, in the bicuspid and molar regions apposition was good.

NotE. In shrapnel injuries of the jaw, it is not often that the wound is clean enough for wiring or a plastic operation or both, to be done so soon. The question arises as to whether, in this case, it would not have been better to have left the gap in the left lower jaw until later, when a prothesis might have assured him an accurate bite.

\section{Larynx.}

No. 26. Pte. M. (CApt. Mumford's Patient).

Whether shrapnel or bullet unknown. Shot transversely across thyro-hyoid space. Entrance : right side. Exit: left. Enormous œdema of all soft tissues. On admission, urgent dyspnœa, relieved by instant high tracheotomy. So great was the œdema that the trachea was not encountered until the soft tissues had been divided to a depth of more than two inches. The body of the hyoid bone was in fragments. The epiglottis was found severed half an inch above its base. The alæ of the thyroid were found divided between the false and true cords. The stump of the epiglottis, with the great cornua of the hyoid bone, and the upper two-thirds of the divided thyroid alæ, were pulled up by the muscles attached to them; 
and the lower thirds of the thyroid alæ were pulled down and so tilted that the rima glottidis faced almost directly forwards. The hyo-glossus and geniohyo-glossus muscles, though not recognizable, must have been severed, for a finger in the mouth and another in the wound were separated by only the dorsal epithelium of the tongue. The enormous œdema made an œsophageal tube impassable ; and there was no wound in the pharyngeal wall through which to pass one. Rather than add to the destruction of parts by making a pharyngeal opening for a tube, through utterly septic tissues, it was decided to perform a temporary gastrostomy on the next day, when the patient would have somewhat recovered from shock. In twenty-four hours, however, after diligent fomenting and cleansing, the œdema had so far subsided, that an œsophageal tube was passable through the mouth. The head was kept continuously flexed day and night. On the ninth day the patient could whisper. Swallowing was possible but difficult, and always resulted in regurgitation into the larynx and then out through the tracheotomy wound. On the twentieth day he was sent home to the care of specialists.

\section{Jaw.}

Shrapnel.

No. 27. Sergt. S.

Skiagram. Mid-part of lower jaw, carrying all four incisors, was comminuted. There was another fracture just in front of right angle of jaw. The mouth and wounds were extremely septic.

Operation (with Dr. Valadier of Paris). Various teeth, small sequestra, and a large central comminuted segment of lower jaw, removed. Mucous membrane and lower lip repaired. Drainage into mouth from beneath chin. Sent home for a prothesis at a later date. 


\section{I34 INJURIES OF NOSE AND THROAT}

\section{No. 28. LCE.-Corp. P.}

Shrapnel. Admitted with an enormous dirty wound of lower part of face. Soft parts : on left side the whole of the floor of mouth was herniated downwards and outwards to the mylo-hyoid region; there was a gap from angle of the mouth to below the chin, and another in the left cheek, through which the masseter muscle was herniated; the lower lateral incisor was projecting through the skin of chin. The anterior quarter of the tongue had been shot off. Patient could not speak or whisper.

Skiagram. No fragments of shrapnel seen. On the left side the outer wall of the left antrum was fragmentary, and the whole of the horizontal ramus in several pieces, completely rotated on its long axis, so that the contained teeth pointed downwards. On the right side, the horizontal ramus was comminuted. The mid-part of both superior maxillæ (pre-maxillary bones), carrying the four incisor teeth, had been blown out.

Operation. The broken outer wall of the left antrum was removed by combined Caldwell-Luc and external routes. On the right side the bone of the lower jaw was'found finely comminuted ; on the left, the pieces were larger, but obviously necrotic. Accordingly the jaw from angle to angle was removed. The left masseter was replaced. Plastic operation on lip, face, and floor of mouth. Drainage by a tube with lateral holes, transversely through sub-maxillary regions and floor of mouth. Jaw-bandage to hold up the sagging soft parts below the mouth and chin. Subsequent history: great œedema persisted for several days, but yielded to fomentations and irrigation. $\mathrm{He}$ was sent home for a prothesis. 


\section{No. 29. PTE. B.}

Shrapnel. Entrance: two inches below angle of left jaw. No exit wound.

Skiagram. Showed two fractures in lower jaw: (I) simple, in middle line ; (2) compound, in front of left lower molar. Shrapnel was seen in the left jaw opposite the first pre-molar.

There was no paralysis of the tongue on either side. Larynx normal. The wound had been accompanied with hardly any bleeding.

Operation. Removal of shrapnel, dead bone, and teeth, by the mouth.

NotE. The superior laryngeal and hypoglossal nerves and the lingual artery were evidently missed by the projectile.

\section{No. 3o. Pte. B.}

Shrapnel. There was an enormous chasm in the lower part of the face. Above, both pre-maxillæ and other contained teeth were gone; the right upper alveolus was separated from the hard palate, and dislocated outwards. From here down to the upper margin of the thyroid cartilage, there was merely a hole, with the soft parts hanging out in shreds, so that pieces of muscle, tendon, nerves or blood-vessels could not be identified. The hard and soft palates were split longitudinally in their whole extent, leaving a large gap into the nose. There was no columella or upper lip. Laterally, the extent of the wound was from one inch in front of the left tragus, to half-way between the right ear and angle of the mouth.

Both eyes were black; there was a triangular hæmorrhage on the outer side of the right conjunctiva. There was no epistaxis or rhinorrhœa.

The whole wounded part was black and gangrenous.

T. 99.4. P. I20, very thin. R. 30 . 
Treatment. Polyvalent anti-streptococcic serum and anti-tetanic serum, every other day. Intravenous infusions. Hypertonic saline dressings in the intervals of constant fomentations. For three days he was fed by a nasal tube, after that by the hole where his mouth had been. On the third day there was a sharp hæmorrhage from the left coronary artery, which was tied. He died one week after admission.

Note. This case is the worst of the series of pharynx and jaw injuries here reported. It is remarkable that important structures, such as the great vessels of the neck, were not so injured as to cause almost immediate death.

\section{Dislocated Pre-maxillae.}

\section{No. 3I. PTE. D. (Fig. 9.)}

Bullet. Entrance : just in front of centre of anterior border of vertical ramus of right upper jaw. Exit : through mouth, tearing the upper lip slightly, just to the left of the middle line. Movements of lower jaw quite free. As regards apposition of teeth, the upper incisors and canines closed three-quarters of an inch in front of the lower. Laterally there was normal apposition of the bicuspids and molars on the left, but on the right the lower jaw teeth closed threequarters of an inch outside the upper jaw teeth.

Skiagram. Complete separation of the pre-maxillæ, which, with their intact contained teeth, projected horizontally forwards. No damage to lower jaw.

Treatment. Four days were devoted to cleansing the mouth. On the third day there was a sharp hæmorrhage from the left coronary artery, which was ligatured.

Operation. On the fourth day (with Dr. Valadier of Paris), the upper right alveolus was found dislocated 

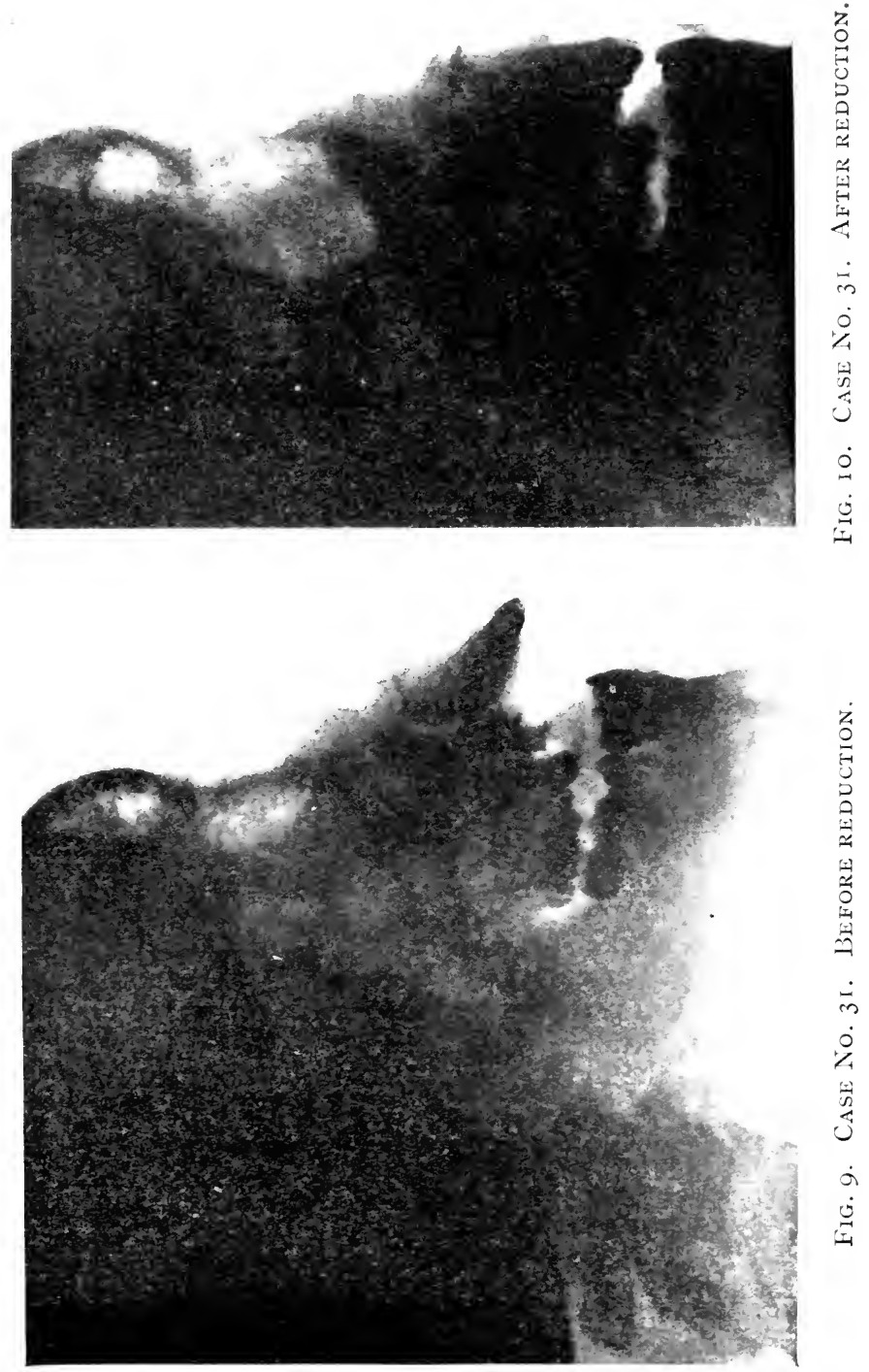


\section{I38 INJURIES OF NOSE AND THROAT}

inwards on to the palate. It was reduced, and remained in position. The dislocation of the premaxillæ was reduced, and kept in position by a silk ligature from the incisor to the first molar on each side. Drainage-tube through the right cheek wound into the mouth. A rent in the dorsum of the tongue was sutured. Sent home well. (Fig. Io.)

\section{Spheno-maxillary Fossa.}

No. 32. Corp. W. (CAPt. Clementi-Smith's Patient).

Shrapnel. There was a vertical gap, one inch wide, from below the right inner canthus to the right angle of the mouth. The whole of the right upper jaw, except the orbital floor, was missing, with its teeth. It was easy to look directly into the right sphenomaxillary fossa. Nose : the lower two-thirds of the outer wall, with the inferior turbinate, and the lower quarter of the septum, had been shot away.

Skiagram. No shrapnel seen.

Treatment. The two weeks following the injury were devoted to cleansing.

Operation. The right cheek was joined on to the nose, and a plastic operation done to repair the upper lip. When he was sent home to have a dental plate fitted, five weeks after the wound, the scar was becoming less perceptible, and he could speak quite well (except dental and palatal consonants), but the left angle of the mouth remained distinctly depressed.

\section{Jaw.}

\section{No. 33. Sergt. S.}

Shrapnel. Entrance: at right angle of mouth. Exit: 2 inches below the centre of the horizontal ramus of the right mandible. Right lower lip torn through to chin. 
Skiagram. No shrapnel seen. On the right side, a vertical fracture through the middle of the horizontal ramus of the lower jaw. On the left side, the premaxilla, destitute of teeth, was dislocated forward.

Operation (with Dr. Valadier). Dead teeth and bone removed. Left pre-maxilla reduced. To keep it in position, a small hole was drilled through the alveolus, as near as possible to its free edge ; through this, a ligature was passed, and anchored round the last molar tooth on the same side. Mucous membrane over the alveolus was repaired. On the right side the posterior half of the horizontal ramus was found dislocated outwards and rotated outwards, and when reduced would not stand in position. This was inaccessible for wiring through the entrance wound, and it was decided to wait some days, until the parts should be quite clean, before wiring it through an external incision. Plastic operation to repair lip and cheek.

\section{Larynx.}

\section{No. 34. PTE. D.}

Bullet. Traversed sub-glottic space from left to right, simultaneously damaging the upper opening of the osophagus. Voice reduced to a whisper. Regurgitation of food, solid or liquid. Tracheotomy was performed without delay.

Larynx. The aryepiglottic folds and false cords were œdematous, narrowing the antero-posterior length of the false glottis. The laryngeal box, as a whole, was rotated on an antero-posterior axis, so that the glottic opening faced slightly to the right.

Thus, the right true cord remained just visible. The left was quite hidden by being tilted bodily outwards beneath the over-lying œdematous false cord.

This condition of things at first conveyed two false impressions, namely, that the adduction of the left 
cord was impaired, and that the right cord was at a lower level than the left (suggesting a lesion of one crico-thyroid muscle).

As the œdema subsided, a fairly strong, rough voice was produced whenever the orifice of the tracheotomy tube was closed by a finger.

Note. The two features simulated in this case, actually occurred in Case No. 35, with which it should be compared.

\section{No. 35. Pte. D.}

Bullet. From left to right, traversing the middle of the thyroid cartilage. Voice reduced to a whisper. Phonatory waste of air. No dyspnœa.

Larynx. Slight limitation of adduction and abduction of the left cord. The right cord slightly over-adducts to compensate. Cords apparently shortened owing to œdema. In adduction, the left cord lies $\frac{1}{8}$ inch below the right. There was faint crenation of the edges of both cords. The pyriform fossæ and arytenoids were œdematous. Externally, the tissues over the thyroid cartilage were tender and widened from side to side (perichondritis).

Diagnosis. One or more of the following structures had evidently sustained injury on the left side : the vagus nerve, or its superior laryngeal branch, or else the crico-thyroid muscle.

The first possibility was negatived by the absence of any other vagus lesion (heart, respiration), or of damage to the other contents of the carotid sheath; the second alternative was eliminated by finding that there was no hemi-anæsthesia of the larynx. It was, therefore, concluded that the crico-thyroid muscle had been damaged.

Note. Contrast Case 34 . 


\title{
Functional Aphonia.
}

\author{
No. 36. PTE. D.
}

No wound. Sudden complete loss of voice in the trenches.

Larynx. During ordinary respiration, was normal. On being told to say ' a ' the cords adducted symmetrically and for their whole length, as in a normal person; but as soon as their edges met, the patient ceased to expire, and of course no sound was produced. There was not, as in typical hysterical aphonia, any diamond-shaped space between the cords ; adductors and tensors were in perfect working order. Confirmatory of the obviously hysterical nature of the condition, it was found that the patient could not even whisper.

Subsequent history. A good prognosis was given, and next day the patient recovered his voice as suddenly as he had lost it.

\section{Foreign Body in Esophagus.}

No. 37. Gunner S. (Fig. II.)

While drinking the gravy out of a bully-beef tin, in the trenches, he swallowed a circular piece of tin, which was lying at the bottom, in the gravy. The piece swallowed was larger than half-a-crown, somewhat bent, and jagged at the edges. He came under observation eight days later.

Skiagram. Showed the foreign body impacted in the coronal plane opposite vertebra $D_{1}$.

Operation. As his occupation in peace time was to play the oboe, it was desirable to avoid tracheotomy or laryngotomy. The anæsthetic was therefore given on an ordinary mask. The œsophagus was exposed in the left posterior triangle of the neck and opened. The foreign body had slipped down one inch lower 


\section{I42 INJURIES OF NOSE AND THROAT}

than the skiagram showed it. The œsophageal mucous membrane was found to be caught in and folded round its jagged edges, making extraction very difficult. After its removal, an œsophageal tube was passed through the mouth; the patient was subsequently taught to introduce this tube for himself,

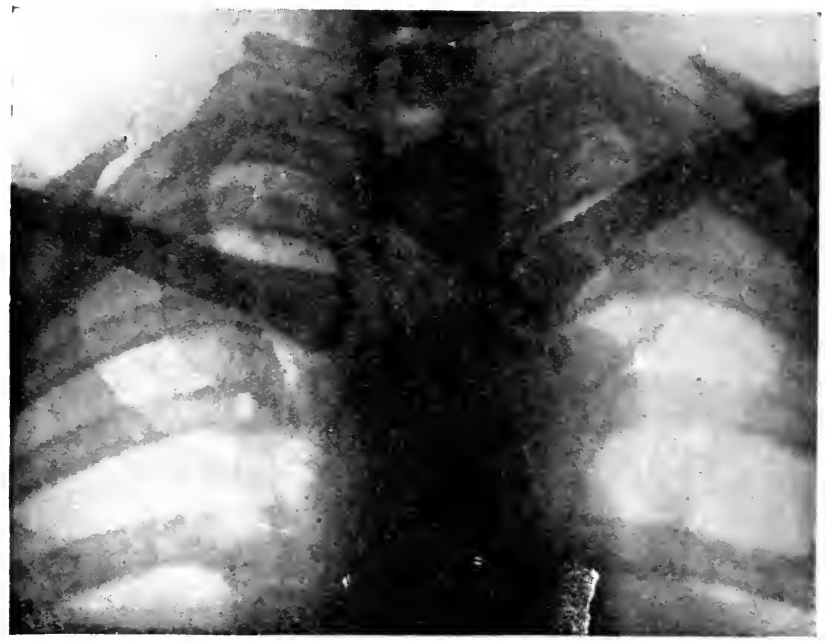

Fig. il. Case No. 37.

for ten days, for feeding purposes. The osophagus was closed and the outer wound packed. At the operation the cervical sympathetic cord and its middle ganglion were clearly exposed, and at several dressings the left pupil was observed to dilate when ribbon gauze was being packed between the osophagus and spine. Recovery was uneventful, and two months after the operation the linear scar was not noticeable when the patient was ordinarily clad. 
Note. This case, which was exhibited at the Roy. Soc. Med. on February 5, I9I5, illustrates the advantages of selecting the posterior triangle of the neck for the operation of osophagotomy.

\section{Trachea.}

\section{No. 38. Pte. H.}

Shrapnel. There was an entrance wound in the suprasternal notch, at the exact point where a low tracheotomy would be done. His voice was hoarse. He had spat up blood until admission, when this ceased. Ever since receiving the wound he had had pain in the lower part of the left axilla.

Skiagram. In the middle of the left lung there was an irregular cavity, measuring 2 inches vertically by 3 inches transversely. In the outer part of this cavity, $I_{2} \frac{1}{2}$ inches from the axillary surface of the sixth left rib, was seen the shrapnel-casing.

Lungs (Captain T. R. Elliott). At the left base, in front, there was deficient movement, and behind the breath sounds were weak. Vocal fremitus and vocal resonance were lost. There was no œgophony.

Further procedure. It was necessary to know precisely the extent of damage suffered by the trachea, partly in order to gauge the probability of dangerous dyspnœa, and also-and more immediately - to determine whether the patient could safely tolerate deep and prolonged anæsthesia for the removal of the shrapnel from the lung.

Accordingly the patient was given $\frac{1}{2}$ grain of morphine and $\frac{1}{10}$ of atropine, very lightly anæsthetized with chloroform, and a tracheoscopy tube passed. There was no visible solution in continuity of the tracheal rings, the only abnormal sign being redness and swelling of the mucosa. It was therefore 


\section{I44 INJURIES OF NOSE AND THROAT}

obvious that the shrapnel must have passed sideways in front of the trachea without perforating it, and that for any necessary interference with the lung, a long general anæsthetic would be perfectly safe.

Note. This case illustrates the value of endoscopic methods in war. 


\title{
INJURIES OF THE EARS
}

\author{
BY
}

\section{CHARLES ERNEST WEST, F.R.C.S. (ENG.)}

AURAL SURGEON TO AND LECTURER ON AURAL SURGERY AT ST. BARTHOLOMEW'S HOSPITAL; CAPTAIN R.A.M.C. (T.F.) 



\section{INJURIES OF THE EARS}

INJURIES of the ears may be best considered on an anatomical basis, it being of course evident that an individual injury may involve several of the subdivisions under which the ear is considered.

The Pinna.-Considering its exposed position the pinna appears to suffer very rarely. The injuries it receives are direct, and are of the nature of torn wounds or loss of substance, and occasionally bruises with production of hæmatoma. Where the injury is confined to the pinna and does not extend to the margins of the external auditory meatus the resultant damage is an æsthetic one only. Serious deformity of the pinna is, however, so conspicuous and unsightly that every effort should be given to securing good repair where possible. The blood supply of the pinna is abundant, and while any base of attachment to the head is left there is good hope of the survival of an almost completely separated ear. In lacerated wounds no voluntary sacrifice of tissue should ever be allowed. On the other hand, the form of the external ear depends entirely on its cartilaginous framework, and once infection of the perichondrium has commenced, its extension, with subsequent necrosis of cartilage and great deformity, is probable.

Lacerations and wounds of the pinna must be treated as individual problems. Linear wounds 
should be sutured and gaps should be drawn together as far as may be. Sutures should not penetrate the perichondrium, but should be through skin only. Perichondritis is best treated by hot fomentations. Unless there is actual suppuration it should never be incised, but an effort may be made to diminish tension by aseptic puncture. In civil practice the majority of cases of perichondritis of the pinna are due to infection with $B$. pyocyaneus, and the administration of a vaccine of this organism has seemed in the writer's experience to favourably modify the course of what is at best a tedious and disappointing condition.

\section{The External Auditory Meatus.-Wounds of the} external meatus vary widely in their character as they involve either the cartilaginous or the bony meatus.

Wounds of the cartilaginous part are direct. Where they result from the tearing away of the pinna they involve as a rule a large segment of the circumference of the meatus and are very apt to result in serious cicatricial contraction of the lumen in healing. Very little can be done to prevent this, but sutures should be inserted whenever possible with the object of minimizing the raw surface and securing early healing. When serious contraction has taken place it is best treated by a planned plastic operation when healing is sound. In two cases the writer has seen wounds of the anterior wall of the cartilaginous meatus by a rifle bullet entering obliquely from behind. A small wound of entry resulted which gave no serious trouble, though naturally the further course of the bullet had done grave damage. 
Wounds of the bony part may be either direct or indirect as a part of an extensive fracture of the skull. Direct wounds which are more or less in the axis of the meatus will generally be immediately fatal, though it is possible for a spent bullet to lodge in the petrous through the external meatus. Direct wounds in a direction transverse to the meatus may shatter its walls without penetration of the cranium.

Such wounds may involve the mastoid behind, and the temporo-maxillary joint in front, with extension to the pharynx, nose, or upper jaw or orbit of the same or crossed side. These wounds are always serious, but especially so when they are complicated by antecedent middle-ear suppuration. In nearly all cases they will require a plastic operation on the meatus, and where the middle ear has been disorganized or is already the seat of chronic infection it is well to incorporate this in a radical mastoid operation. By this means the whole area is drained and the temporomaxillary joint is rendered freely accessible. Fractures of the roof of the external meatus occurring as a part of an extensive fracture of the skull are usually compound by tearing of the lining cuticle of the meatus. They are in themselves rarely of importance except as placing the diagnosis of basal fracture on a secure basis. The membrana tympani and roof of the middle ear are usually also involved. Only in the presence of an infected tympanum do they assume any serious importance. The external meatus should be dried, filled with boracic powder, and closed by a dry dressing, except in the latter case. Unless 
there is evidence of meningitis, an absolutely expectant treatment is wise. The ear should never be syringed out, but in the presence of discharge the meatus should be frequently wiped out and refilled with boracic powder.

\section{Membrana Tympani and Tympanic Structures.-}

Rupture of the membrane is a not very uncommon war-injury, resulting generally from the explosion of heavy shell close to the sufferer. The tears seen due to this cause have been much less regular in their shape and position than those familiar in civil life. They are apt to be extensive and may give the impression of actual loss of substance. The immediate results are bleeding from the ear in small amount followed by a serous discharge. There is deafness and frequently some vertigo. These points will be dealt with later. In extreme cases there may be disorganization of the tympanic structures with dislocation of the malleo-incudal joint. The external meatus should be wiped out and dried, powdered, and lightly plugged with gauze. After this, as a rule, the more the ear is left alone the better. The prognosis for hearing is generally good. (See further: 'Deafness'.)

Direct wounds of the walls of the tympanic cavity do not need to be considered as isolated lesions. They necessarily involve other important structures. The more immediate of these will be, internally, the labyrinth, the internal auditory meatus, and the pons : inferiorly, the vault of the jugular bulb: posteriorly, the facial nerve, the mastoid region, and lateral sinus: anteriorly, the internal carotid artery. When the 
patient is alive the only urgent need is likely to be the control of hæmorrhage. When this is venous, any way of stopping the external meatus will suffice for the time. Later, the cavity of the wound must be carefully and deeply plugged with gauze, which should if possible be left undisturbed for at least forty-eight hours. Where bleeding is from the internal carotid, the only chance for the patient lies in control of the artery in the neck until it can be ligatured in this part. The writer was able to treat successfully in this way the only case of bleeding from the intrapetrous carotid which he has seen. No reliance should be placed on the cessation of bleeding after pressure. As soon as the blood-pressure rises again, hæmorrhage will recur. In spite of the basal anastomosis, no hæmorrhage took place from the distal end of the vessel in the case mentioned.

The Bony Labyrinth.-Gross mechanical damage to the labyrinth may be due to direct injury by a projectile or to the spread of a fracture through the petrous. The commonest fracture to involve the petrous in this way is one produced by violence applied to the opposite parieto-occipital region, passing thence downwards across the foramen magnum and the opposite jugular fossa, and dividing the petrous in a plane vertical to its posterior and upper surfaces. The fracture passes right through the in: ternal ear, often completely severs the facial nerve close to the geniculate ganglion, and having entered the middle ear, either continues outwards along the roof of the external meatus or turns forwards along 
the line of the roof of the Eustachian tube. In either case the damage to the labyrinth as a functional organ is complete and irreparable. There will be total deafness, vertigo, and, if the patient survive, a more or less prolonged period of incoordination. The immediate surgical danger is of meningitis by extension of infection from the opened middle ear along the line of the internal auditory meatus and so to the cisterna pontis. The external meatus should be carefully cleaned by swabbing with sterile wool and painted with iodine. A wide area round the entrance of the external meatus should be rendered sterile, and abundant dressings applied. No effort should be made by plugging to interfere in any way with the free flow of cerebro-spinal fluid. If the ear has been the seat of suppuration and the condition of the patient justifies it, the safest treatment is probably by a radical mastoid operation with free opening of the outer wall of the labyrinth from the middle ear. The escape of cerebro-spinal fluid may be very free, necessitating frequent packings and renewal of the outside parts of the dressing. It persists for from two or three days to a fortnight, rarely longer, and comes to an end rather suddenly. After two days' cessation of the flow with absence of meningeal symptoms the patient may be considered safe from this danger.

The Mastoid.-Wounds involving the mastoid region in more than the most superficial fashion almost necessarily extend beyond it either to the interior of the cranium, the neck, or the pharynx and 
post-facial region. The local damage in the mastoid is thus of only secondary importance. The cellular character of the bone in this part may lead to troublesome chronic suppuration or may even produce an acute infective mastoiditis. If infection of cellular bone has taken place, the whole of the cellular area should be opened up and free drainage secured. Under these conditions no further local trouble is likely to occur. Where drainage is deficient, infection may spread to the lateral sinus, the cerebellum, the meninges, or the deep planes of the neck. Such extensions must be treated on general surgical principles according to the indications of the individual case.

Deafness ascribed to war-causes presents varied features and may be due to any one of a number of conditions, meatal, tympanic, labyrinthine, or central. The most typical condition during the war has been due to the explosion of shell. During the earlier months of the war such cases were much more frequent than they have appeared to be during the present year. In making the diagnosis a careful history should be taken, but the conclusions arrived at should not be influenced by this in the event of its being in discord with the hearing tests. In the writer's experience nearly everything is put down by the patient to explosion, often erroneously. It should be remembered that many deafnesses go undiscovered until the patient's attention is directed to them.

Meatal obstructions by ceruminous plugs are not uncommon. It seems likely that some of these are truly ' explosion deafnesses' owing to the plug having 
been driven home by the sudden compression-wave. Such plugging of the meatus seems to give complete immunity from damage to the cochlea, and the case is cured by the syringe. These cases have, however, repeatedly been sent home from France for want of a simple diagnosis. The deafness is purely of the obstructive type. A few cases have been seen in which the swelling due to a meatal furuncle has been responsible for deafness, again of course of the 'obstructive' type.

Tympanic conditions producing an 'obstructive' deafness are common. In truly recent cases they include simple Eustachian catarrhs, due to the spread of a nasopharyngeal 'cold', and all degrees of tympanic inflammation from a mild catarrh to an acute otitis media. The diagnosis between them must be made on the otoscopic appearances. In addition there will be met numerous cases in which there is evidence of a long-standing middle-ear suppuration. In some of these the deafness has been truly discovered, in others it has probably been made the excuse for a rest. The treatment of all these conditions presents nothing beyond the routine of civil practice. It is remarkable with what completeness the possession of an antecedent middle-ear deafness seems to screen the labyrinth from damage by explosion. While the writer has met numerous cases of alleged explosion-deafness which have proved to have old perforations, he has only been able to substantiate the presence of a cochlear deafness in one such case. The deafness due to rupture of the membrane and swelling of the tympanic 
mucosa is often very great and accompanied by pulsating and buzzing noises. The prognosis is generally good, the deafness being mainly or entirely due to the middle-ear condition and improving pari passu with this.

The diagnosis between 'obstructive' deafnesses and those of labyrinthine type is simple. It rests on the presence or absence of serious impairment of perception of the tuning-fork when firmly applied to the mastoid, and though the specialist will confirm and amplify this observation by a variety of further tests, this basis may be accepted as sufficient for the general purposes of this book. If perception through the bone is intact, the deafness is not due to a labyrinthine or central cause. On the other hand, the determination between a true labyrinthine deafness and one of central origin is extremely difficult. A few cases of syphilitic deafness have been met; the possibility of this cause must not be lost sight of in considering a case of labyrinthine deafness.

Explosion-deafnesses need no active treatment. Compared with the ordinary 'gunner's deafness' their prognosis is favourable, and the majority of them improve greatly with time. The worst cases remain totally or almost totally deaf; the lightest recover so completely that no loss of hearing is obvious. In many there is undoubtedly a central factor of 'nervous shock', and some of these recover completely after a few days' rest and quiet. Where tinnitus is troublesome, hydrobromic acid may be freely used for a time. The condition is rarely 
obstinate. Where there is a true associated vertigo, it is probable that hæmorrhage has taken place into the labyrinth, and the outlook is less favourable. Above all things deafness of this class should not be submitted to vibratory treatments or the so-called oto-massage. 


\section{INDEX}

Amblyopia, 62.

functional, 62 .

Antrum of Highmore, operations on, 86.

of Highmore, wounds of, 85 . cases of wounded, I I 8 , I 2 I, I 24, I 25, I 26, I 27, I 34 .

Aphonia, functional, I4I.

Aqueous humour, treatment of blood-clot in, $2 \mathrm{I}$.

Auditory meatus, wounds of, I 48 .

Blindness, traumatic, I2.

Bullet wound of eye, case of, I 7 .

Burns of eye, 46.

Cases illustrative of wounds of the nose and throat, I $6-44$.

Choroid, hæmorrhage from, I7.

Ciliary body, hæmorrhage from, 17 .

Collargol, use of, 30, $3 \mathrm{I}$.

Corneal wounds, 24 .

Deafness, I 53 .

diagnosis of, I 55 .

'explosion', I 53, I 55 .

'gunner's', I 55 .

labyrinthine, I 53,155 .

meatal, 153 .

obstructive, I 54 , I 55 .

Ear, wounds of external, 147. Ears, injuries of, I 47-I 56.
Ethmoid, case of wounded, I 26.

Explosion deafness, I 53. injuries to eye, 46,50 .

Eye, burns of, 46 .

explosion injuries of, 50 .

injuries of, 8-65.

sympathetic inflammation of, 52-6.

sympathetic irritation of, 54.

Eyeball, evisceration of, 3I. examination of, for foreign bodies in, 34 .

foreign bodies in, 33 .

incised wounds of, 24 .

injuries to, I I.

penetrating wounds of, 23 , 36.

removal of foreign bodies from, 34,38 .

after treatment of, 35 . rupture of, treatment of, $20,22$.

sequelæ of wounds of, 43 . treatment of injuries to, 20.

Eyelid, injuries to, I I.

Foreign bodies in the eyeball, method of examining for, 34 .

method of removal, 34 , 38 .

Fractured jaw, cases of, I29, I 30, I 3 I, I 33, I 35, I 38 .

Frontal sinus, cases of wounded, I28, I 30, I 3 I. 
Functional amblyopia, 62 . aphonia, I4I.

Globe of eye : degenerative changes after wounds of, 43 .

evisceration of, $3 \mathrm{I}$. examination of, for foreign

bodies, 34 .

incised wounds of, 24 .

injuries to, I 4 .

penetrating wounds of, 23 , 36 .

removal of foreign bodies

from, 34,38 .

rupture of, treatment of, $20,22$.

treatment of injuries to, 20 .

'Gunner's' deafness, I 55.

Hæmorrhage from choroid, I 7 . from ciliary body, 17 .

from iris, 17 .

from wounds of throat, I I I . intra-ocular, 16.

retinal, 18 .

sub-conjunctival, I 5 .

Hard palate, cases of wounded, I 20, I 29, I 35 .

Head injuries, ocular symptoms accompanying, 64 .

Illustrative cases of wounds of the nose and throat, I I6-44.

Injuries to ears, $147-56$.

to eye, 8-65.

to eyeball, I4.

to eyelids, I I.

to globe of eye, I 4.

to orbit, I 2.

Inflammation, sympathetic, of eye, 56 .

Intra-ocular hæmorrhage, I6. Iris, hæmorrhage from, I 6. prolapse of, treatment of, 25.
Irritation, sympathetic, of eye, 54 .

Labyrinth of ear, wounds of, I $5 \mathrm{I}$.

Labyrinthine deafness, I 53, I 55 .

Laryngotomy, I I I .

Larynx, cases of wounded, I 32, I 39, 140 .

diagnosis of wounds of, 107.

effect of nerve injury on, IO3.

foreign bodies in, 99.

perforating wounds of, IOI. treatment of wounds of, I IO, I I 2 .

Lens dislocated, treatment of, I9, 21 .

wounded, treatment of, 27 , 29,30 .

Mastoid region, wounds of, I 52 .

Maxilla, cases of wounded, I 29, I 30, I 3 I, I 33, I 35, I 38 .

Maxillary sinus, wounds of, 85.

Meatal deafness, I 53.

Meatus auditorius, external, wounds of, I 48 .

Membrana tympani, wounds of, 150 .

Nasal fossa : anatomy of, 73 . wounds of, 83 .

Nasal septum, anatomy of. 74 .

Nasal sinuses : anatomy of, 75 . wounds of, 88 .

Neck, anatomy of, 90 . operations on, 94.

Nose, anatomy of, 7 I. 
Nose and throat, injuries of, 69-I 44 . fracture of, 78 . plastic surgery of, 82 . traumatic deformities of, 79. wounds of, $77,81,116$.

Obstructive deafness, I 54, I 55 . Ocular hæmorrhage, I 5 .

Esophagotomy, case of, 142. Esophagus, foreign body in, case of, I4I.

treatment of wounds of, I I 3 .

Omnopon-scopolamine, use of, 29.

Ophthalmia, sympathetic, 52 . Ophthalmoplegia, traumatic, I 3 .

Orbit, injuries to, 12.

Palate, hard, cases of wounded, I 20, I29, I 35 .

Paraffin as a prothetic, 32 .

Pharynx, treatment of wounds of, II 3 .

Pinna of ear, wounds of, 147 .

Pre-maxillæ, case of dislocated, 136.

Retina, detachment of, treatment of, 19, 2 I.

Retinal hæmorrhage, I8.

Rupture of eyeball, 20.

Sclerotic, wounds of, 27.

Septum nasi :

anatomy of, 74 .

case of wounded, II6.

Sinuses, nasal, anatomy of, 75 . Spheno-maxillary fossa, case of wounded, 138 .

Symblepharon, treatment of, 49.

Sympathetic inflammation of eye, 56 .
Sympathetic irritation of eye, 54. ophthalmia, 52 .

Throat, anatomy of, 90 . and nose, injuries of, 69I 44 . effects of wounds of, 97 . operations on, 94.

treatment of wounds of,

I IO-I 2 .

wounds of, 90-I 44 .

Trachea, case of wounded, 143 .

Tracheotomy, I I I .

Treatment of infected wounds of eye, 29. injuries to globe of eye, 20 . simple wounds of eye, 28 . wounds of larynx, I IO, I I 2. wounds of lens, 30 . wounds of œsophagus, I I 3 . wounds of pharynx, I 3 . wounds of throat, I IO, I I 2. Tympanum, wounds of, I 50 .

Vocal cords, paralysis of, 97, I04, I 09.

Wounds of

antrum, cases of, I I 8 , I $2 \mathrm{r}$, I 24, I 25, I 26, I 27, I 34 .

bony labyrinth of the ear, I $5 \mathrm{I}$.

cornea, 24.

ear, 147 .

external auditory meatus, I 48 .

eye, treatment of infected, 29.

eye,treatment of simple, 28 . eyeball, 23 .

globe of eye, 23 .

hard palate, cases of, I2O, I 29, I 35 . iris, 25 .

larynx, cases of, I32, I39, I 40 . 
Wounds of (continued).

lens, 27.

mastoid region, 152.

membrana tympani, I 50.

nasal septum, cases of,

I 16,125 .

nose, 77 .

nose, cases of, I I6, I I 7, I 23.

œsophagus, treatment of,

I 3 .
Wounds of (continued).

pharynx, treatment of, I I 3. pinna of ear, 147.

sclerotic, 27.

spheno-maxillary fossa, case of, I 38 .

throat, non-penetrating, 98.

throat, penetrating, 99 .

trachea, I 43 . 


\section{OXFORD WAR PRIMERS OF MEDICINE AND SURGERY}

WOUNDS IN WAR: THEIR TREATMENT AND RESULTS.

D'Arcy Power, M.B. (Oxon.), F.R.C.S. (Eng.), LieutenantColonel R.A.M C. (T.) 2s. 6d. net.

SURGERY OF THE HEAD.

L. Bathe Rawling, M.B., B.C. (Cantab.), F.R.C.S. (Eng.), Major R.A.M.C. (T.) 3s. 6d. net.

INJURIES OF JOINTS.

RoBERT Jones, F.R.C.S. (Eng.), Major R.A.M.C. (T.)

GUNSHOT INJURIES OF BONES

3s. 6d. net.

E. W. Hey Groves, F.R.C.S. (Eng.), Captain R.A.M.C. (T.) 3s. 6d. net.

INJURIES OF NERVES.

Purves Stewart, M.D., F.R.C.P. (Lond.), Colonel R.A.M.C. (T.); Arthur H. Evans, F.R.C.S. (Eng.), Captain R.A.M.C. (T.) 3s. 6d. net.

WOUNDS OF THE THORAX IN WAR.

J. KEOGH MURPHY, M.C. (Cantab.), F.R.C.S. 2s. 6d. net. ABDOMINAL INJURIES.

Professor J. Rutherford Morison, F.R.C.S. (Eng.), Hon. Staff-Surgeon R.N.V.R. 2s. 6d. net.

INJURIES OF THE EYES, THROAT, NOSE, AND EARS.

A. Maitland Ramsay, M.D. (Glas.), Major R.A.M.C. (T.); J. Dundas Grant, M.D., F.R.C.S. (Eng.), late Major R.A.M.C. (Post Office Rifle Volunteers); H. Lawson Whale, M.D. (Camb.), F.R.C.S. (Eng.), Capt. R.A.M.C. (T.); C. ERNEST WEST, F.R.C.S. (Eng.), Captain R.A.M.C. (T.) 2s. 6d. net.

NERVE INJURIES AND SHOCK.

WILFRED HARRIS, M.D. (Cantab.), F.R.C.P. (Lond.), Captain R.A.M.C. (T.) 3s. 6d. net.

MEDICAL HINTS.

J. Edward Squire, C.B., M.D., F.R.C.P. (Lond.), late Lieutenant-Colonel (Hon. Colonel) R.A.M.C. (V.) 2s. 6d. net. THE STRETCHER BEARER: A Companion to the R.A.M.C. Training Book.

Georges M. Dupuy, M.D., Stretcher Bearer Ambulance Section (C), Norwood Co., Lambeth Battalion V.T.C. 2s. net.

CEREBRO-SPINAL FEVER.

Thomas J. HoRder, B.Sc., M.D., F.R.C.P. (Lond.), Major R.A.M.C. 3s. 6d. net. 





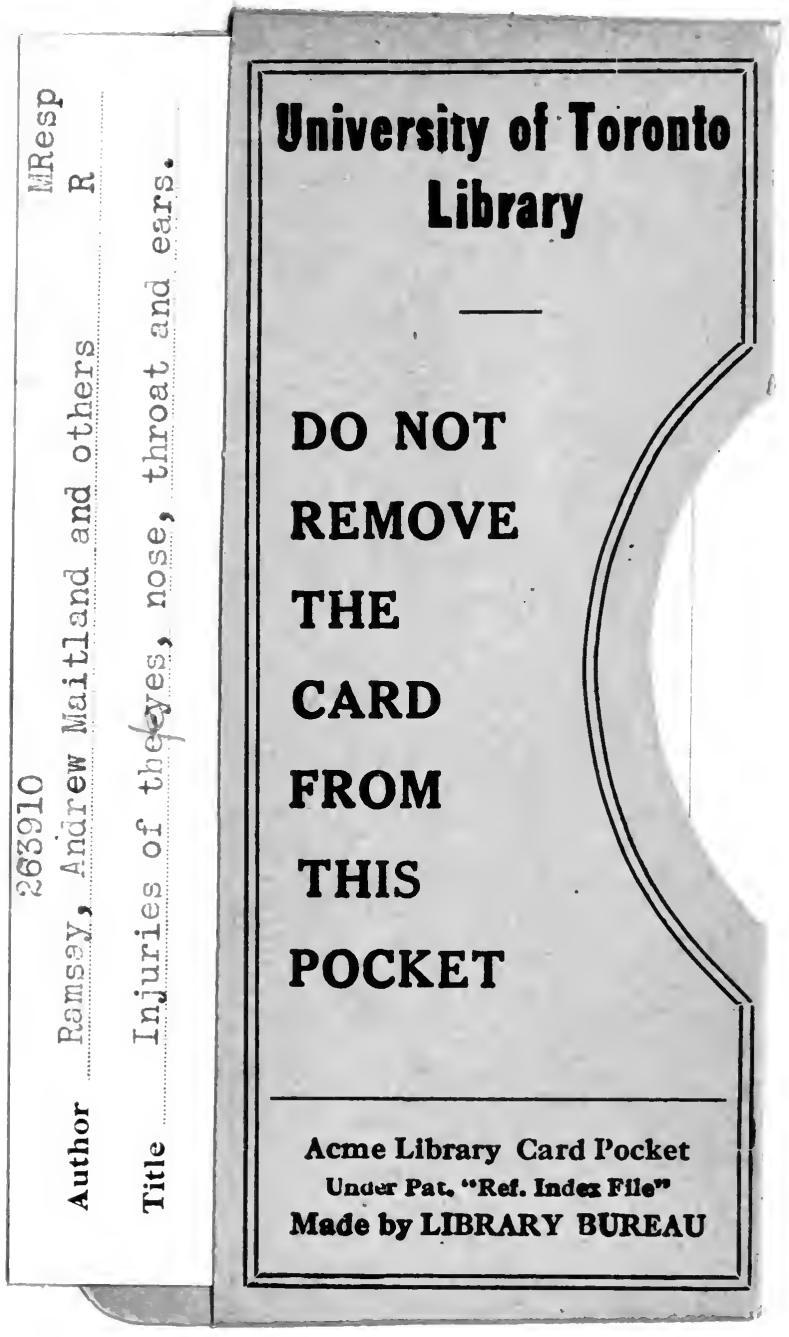




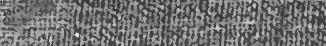

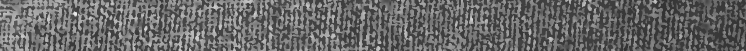

- 3y

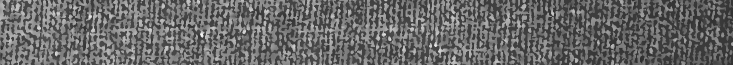
What

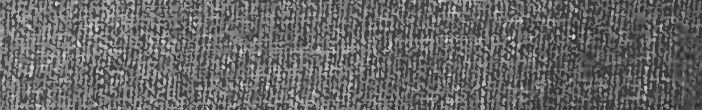

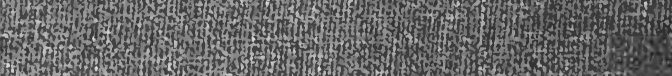

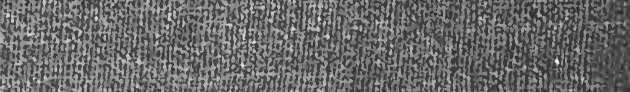

7.

16.

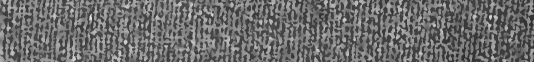

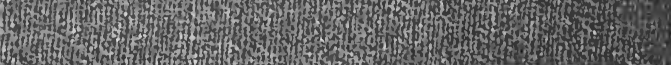

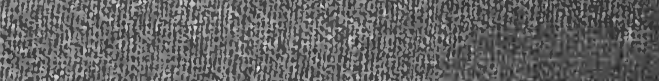

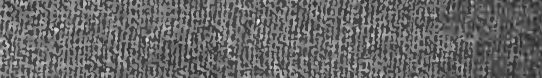

17.

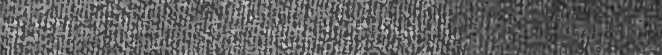

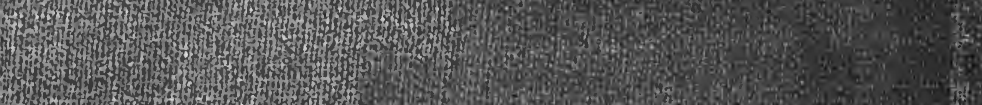

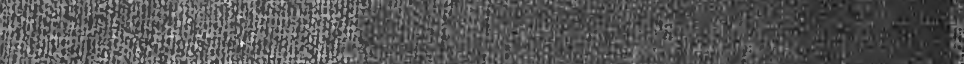

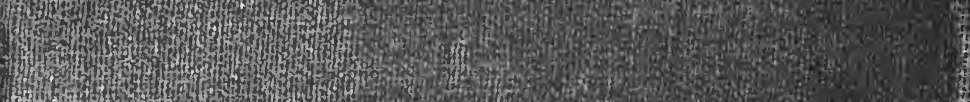

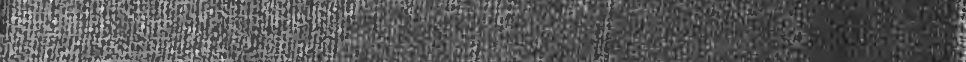

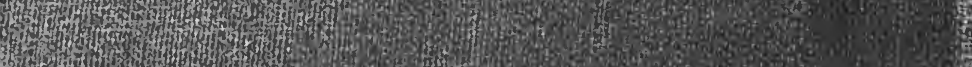

int

H.

W.

s.t.

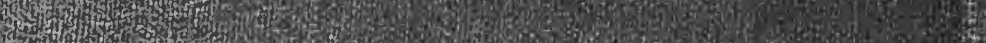

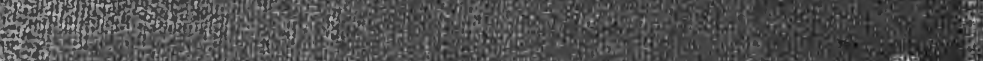

W.

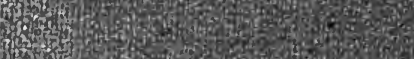

Wring

for

a.

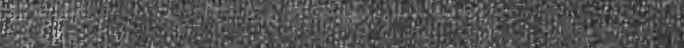

